\title{
UNRAMIFIED COHOMOLOGY OF CLASSIFYING VARIETIES FOR CLASSICAL SIMPLY CONNECTED GROUPS 光
}

\author{
BY ALEXANDER MERKURJEV
}

AbStract. - Let $F$ be a field and $G \subset \mathbf{S L}_{n, F}$ an algebraic closed subgroup of $\mathbf{S L}_{n, F}$. Denote by $B G$ the factor variety $\mathbf{S L}_{n} / G$. The stable $F$-birational type of $B G$ is independent on the choice of an embedding $G \subset \mathbf{S L}_{n}$. The points of $B G$ classify principal homogeneous spaces of $G$. We compute the degree three unramified Galois cohomology with values in $\mathbb{Q} / \mathbb{Z}(2)$ of the function field of $B G$ for all classical semisimple simply connected groups $G$. As an application, examples of groups $G$ (of types $A_{n}$ and $D_{n}$ ) with stably non-rational over $F$ varieties $B G$ are given.

(C) 2002 Éditions scientifiques et médicales Elsevier SAS

RÉSUMÉ. - Soient $F$ un corps et $G \subset \mathbf{S L}_{n, F}$ un sous-groupe algébrique fermé de $\mathbf{S L}_{n, F}$. Notons $B G$ la variété quotient $\mathbf{S L}_{n} / G$. Le type $F$-birationnel stable de $B G$ ne dépend pas du plongement $G \subset \mathbf{S L}_{n}$. Les points de $B G$ classifient les espaces principaux homogènes sous $G$. Pour tout groupe $G$ semi-simple simplement connexe de type classique, nous calculons le troisième groupe de cohomologie non ramifiée, à valeurs dans $\mathbb{Q} / \mathbb{Z}(2)$, du corps des fonctions de $B G$. Cela nous permet de donner des exemples de groupes $G$ (de type $A_{n}$ et de type $D_{n}$ ) pour lesquels $B G$ n'est pas stablement $F$-rationnel.

(C) 2002 Éditions scientifiques et médicales Elsevier SAS

\section{Introduction}

Let $G$ be a (smooth) algebraic group defined over a field $F$. Choose an injective homomorphism $\rho: G \hookrightarrow S=\mathbf{S L}_{n}$ over $F$ and set $X_{\rho}=S / \rho(G)$. We call $X_{\rho}$ a classifying variety of $G$ as $X_{\rho}$ classifies principal homogeneous spaces of $G$ : for every field extension $L / F$ there is a natural bijection [19, Ch. I, §5]

$$
H^{1}(L, G) \simeq X_{\rho}(L) / S(L)
$$

In other words, any principal homogeneous space of $G$ over $L$ is isomorphic to the fiber of the natural morphism $S \rightarrow X_{\rho}$ over some point of $X_{\rho}$ over $L$. The stable birational type of $X_{\rho}$ is independent on the choice of $\rho$; we denote it by $B G$.

We consider stable birational invariants of $B G$, namely, the unramified cohomology defined as follows. For every $d \geqslant 0$ let $H_{\mathrm{nr}}^{d}\left(F\left(X_{\rho}\right)\right)$ be the intersection of the kernels of residue

\footnotetext{
Partially supported by the N.S.F.
} 
homomorphisms

$$
\partial_{v}: H^{d}\left(F\left(X_{\rho}\right), \mathbb{Q} / \mathbb{Z}(d-1)\right) \longrightarrow H^{d-1}(F(v), \mathbb{Q} / \mathbb{Z}(d-2))
$$

for all discrete valuations $v$ on $F\left(X_{\rho}\right)$ over $F$. (Here $\mathbb{Q} / \mathbb{Z}(i)$ is the direct limit of $\mu_{n}^{\otimes i}$ taken over all $n$ prime to the characteristic exponent of $F$.) The group $H_{\mathrm{nr}}^{d}\left(F\left(X_{\rho}\right)\right)$ is independent on the choice of $\rho$ (up to canonical isomorphism) and we denote it by $H_{\mathrm{nr}}^{d}(B G)$. The natural homomorphism

$$
H^{d}(F, \mathbb{Q} / \mathbb{Z}(d-1)) \rightarrow H_{\mathrm{nr}}^{d}(B G)
$$

splits by evaluation at the distinguished point of $B G$, thus,

$$
H_{\mathrm{nr}}^{d}(B G)=H^{d}(F, \mathbb{Q} / \mathbb{Z}(d-1)) \oplus H_{\mathrm{nr}}^{d}(B G)_{\mathrm{norm}}
$$

with the latter group being the group of normalized unramified classes. If the classifying variety $B G$ is stably rational, then $H_{\mathrm{nr}}^{d}(B G)_{\text {norm }}=0$.

The group $H_{\mathrm{nr}}^{1}(B G)_{\text {norm }}$ is trivial. Over an algebraically closed field $F$ the group $H_{\mathrm{nr}}^{2}(B G)$ has been studied in [14,15,2]. Saltman, for $G=\mathbf{P G L}_{n}$, and Bogomolov, for $G$ arbitrary connected reductive, showed that $H_{\mathrm{nr}}^{2}(B G)$ is trivial (see also [4]). In [16] Saltman has shown that $H_{\mathrm{nr}}^{3}(B G)=0$ for $G=\mathbf{P G L}_{n}$ and $n$ odd.

Using [2] (or [4]), one may show that for a (connected) semisimple group $G$ defined over an arbitrary field $F$ the group $H_{\mathrm{nr}}^{2}(B G)_{\text {norm }}$ is trivial. The aim of the paper is to compute the group $H_{\mathrm{nr}}^{3}(B G)_{\text {norm }}$ for any (connected) semisimple simply connected group $G$ of classical type defined over an arbitrary field. The idea is to consider the subgroup $A^{0}\left(X_{\rho}, H^{3}\right)$ of all classes in $H^{3}\left(F\left(X_{\rho}\right), \mathbb{Q} / \mathbb{Z}(2)\right)$ unramified only with respect to discrete valuations associated to irreducible divisors of $X_{\rho}$. This group is also independent of the choice of $\rho$ and we denote it by $A^{0}\left(B G, H^{3}\right)$; thus,

$$
H_{\mathrm{nr}}^{3}(B G) \subset A^{0}\left(B G, H^{3}\right)
$$

Similarly,

$$
A^{0}\left(B G, H^{3}\right)=H^{3}(F, \mathbb{Q} / \mathbb{Z}(2)) \oplus A^{0}\left(B G, H^{3}\right)_{\text {norm }}
$$

where $A^{0}\left(B G, H^{3}\right)_{\text {norm }}$ is the group of normalized classes.

It was noticed by Rost that the group $A^{0}\left(B G, H^{3}\right)$ is canonically isomorphic to the group $\operatorname{Inv}^{3}(G, H)$ of dimension 3 cohomological invariants of $G$, i.e., morphisms of functors

$$
H^{1}(*, G) \longrightarrow H^{3}(*, \mathbb{Q} / \mathbb{Z}(2))
$$

from the category of field extensions of $F$ to the category of sets. The invariants corresponding to the elements of $H_{\mathrm{nr}}^{3}(B G)$ (respectively $A^{0}\left(B G, H^{3}\right)_{\text {norm }}$ ) are called unramified (respectively normalized). The group of normalized invariants $\operatorname{Inv}^{3}(G, H)$ norm has been computed by Rost: if $G$ is absolutely simple simply connected, that group is cyclic with canonical generator $r_{G}$ (called Rost invariant) of certain order $n_{G}^{\prime}$ which can be computed in terms of representation theory of $G$. Thus, in order to compute the group $H_{\mathrm{nr}}^{3}(B G)$ it suffices to determine all multiples $m r_{G}$ of the Rost invariant that are unramified.

It is proved in the paper that if $G$ is simply connected of type $B_{n}$ or $C_{n}$, the unramified group $H_{\mathrm{nr}}^{3}(B G)_{\text {norm }}$ is trivial. On the other hand, for the types $A_{n}$ and $D_{n}$ the group $H_{\mathrm{nr}}^{3}(B G)_{\text {norm }}$ is either zero or cyclic of order 2 and can be determined for all groups in terms of the Tits algebras of $G$. This computation leads to examples of classifying varieties $B G$ that are not stably rational. 
On the other hand, if the Tits algebras of $G$ are trivial (for example, if $G$ is quasi-split or $F$ is separably closed), the group $H_{\mathrm{nr}}^{3}(B G)_{\text {norm }}$ vanishes.

The idea to consider ramification of Rost invariants is due to Rost and Serre (cf. [18]). For the reader's convenience we include proofs of some basic properties of Rost invariants (Appendix A) due to Rost and a computation of Rost numbers $n_{G}$ given in $[9, \S 31]$ without proofs (Appendix B).

\section{Classifying varieties}

\subsection{Definition of classifying varieties}

A connected algebraic group $S$ defined over a field $F$ is called special if $H^{1}(L, S)=1$ for any field extension $L / F$. Examples of special groups are $\mathbf{S L}_{n}, \mathbf{S p}_{2 n}, \mathbf{G L}_{1}(A)$ for a central simple $F$-algebra $A$. Note that the varieties of all these groups are rational.

Let $G$ be an algebraic group over a field $F$. Choose an embedding $\rho: G \hookrightarrow S$ into a special rational group $S$. Consider the variety

$$
X_{\rho}=S / \rho(G)
$$

which is called a classifying variety of $G$. Obviously, $X_{\rho}$ depends on the choice of $\rho$.

Let $\rho^{\prime}: G \rightarrow S^{\prime}$ be another embedding. In order to compare $X_{\rho}$ and $X_{\rho^{\prime}}$ consider the diagonal embedding

$$
\rho^{\prime \prime}=\left(\rho, \rho^{\prime}\right): G \longleftrightarrow S \times S^{\prime},
$$

which induces a surjection $\alpha: X_{\rho^{\prime \prime}} \rightarrow X_{\rho}$. Clearly, $\alpha$ is an $S^{\prime}$-torsor over $X_{\rho}$. Since $S^{\prime}$ is special, this torsor is trivial at the generic point of $X_{\rho}$, hence $F\left(X_{\rho^{\prime \prime}}\right) \simeq F\left(X_{\rho}\right)\left(S^{\prime}\right)$. The group $S^{\prime}$ is rational, so that $X_{\rho}$ is stably birationally equivalent to $X_{\rho^{\prime \prime}}$. Similarly, $X_{\rho^{\prime}}$ is stably birationally equivalent to $X_{\rho^{\prime \prime}}$, hence $X_{\rho}$ and $X_{\rho^{\prime}}$ are stably birationally equivalent. We denote by $B G$ the variety $X_{\rho}$ for some $\rho$. The stable birational type of $B G$ is well defined.

\subsection{Homotopy invariant functors}

Let

$$
J: \text { Fields } / F \longrightarrow \text { Ab }
$$

be a functor from the category of field extensions of $F$ to the category of abelian groups. We say that $J$ is homotopy invariant if for any field extension $L / F$, the map $J(L) \rightarrow J(L(t))$ is an isomorphism.

Proposition 2.1. - Let $J$ be a homotopy invariant functor, $G$ an algebraic group over $F$. Then the group $J\left(F\left(X_{\rho}\right)\right.$ ) depends only on $G$ and does not depend (up to canonical isomorphism) on the choice of an embedding $\rho$.

Proof. - In the notation of (2.1), the field extension $F\left(X_{\rho^{\prime \prime}}\right) / F\left(X_{\rho}\right)$ is purely transcendental, hence the map $J\left(F\left(X_{\rho}\right)\right) \rightarrow J\left(F\left(X_{\rho^{\prime \prime}}\right)\right)$ is an isomorphism. Similarly, we have an isomorphism $J\left(F\left(X_{\rho^{\prime}}\right)\right) \rightarrow J\left(F\left(X_{\rho^{\prime \prime}}\right)\right)$.

We denote the group $J\left(F\left(X_{\rho}\right)\right)$ by $J(B G)$. The group $J(B G)$ can detect stable nonrationality of a classifying variety $X_{\rho}$ : if the natural homomorphism $J(F) \rightarrow J(B G)$ is not an isomorphism, then the variety $X_{\rho}$ is not stably rational. 
Let $\alpha: G \rightarrow G^{\prime}$ be a group homomorphism. Consider two embeddings $\rho: G \hookrightarrow S$ and $\rho^{\prime}: G^{\prime} \hookrightarrow S^{\prime}$ with $S$ and $S^{\prime}$ special rational groups and the embedding $\rho^{\prime \prime}=\left(\rho, \rho^{\prime}\right): G \hookrightarrow S \times S^{\prime}$. The projection $S \times S^{\prime} \rightarrow S^{\prime}$ induces a dominant morphism $X_{\rho^{\prime \prime}} \rightarrow X_{\rho^{\prime}}$ and hence a group homomorphism

$$
J\left(B G^{\prime}\right)=J\left(F\left(X_{\rho^{\prime}}\right)\right) \longrightarrow J\left(F\left(X_{\rho^{\prime \prime}}\right)\right)=J(B G)
$$

for a homotopy invariant functor $J$. Thus, the assignment $G \rightarrow J(B G)$ is a contravariant functor from the category of algebraic groups over $F$ to the category of abelian groups.

\subsection{Cycle modules}

A cycle module $M$ over a field $F$ is an object function $E \mapsto M^{*}(E)$ from the category Fields $/ F$ to the category of $\mathbb{Z}$-graded abelian groups together with some data and rules [13, $\S 2]$. The data include a graded module structure on $M$ under the Milnor ring of $F$, a degree 0 homomorphism $i_{*}: M(E) \rightarrow M(L)$ for any field homomorphism $i: E \rightarrow L$ over $F$, a degree 0 homomorphism (norm map) $j^{*}: M(L) \rightarrow M(E)$ for any finite field homomorphism $j: E \rightarrow L$ over $F$ and also a degree -1 residue homomorphism $\partial_{v}: M(E) \rightarrow M(F(v))$ for a discrete, rank one, valuation $v$ on $E$ over $F$ with residue field $F(v)$.

Example 2.2. - We will be considering the cycle module $H$ given by Galois cohomology [13, Remark 2.5]

$$
H^{d}(E)=H^{d}(E, \mathbb{Q} / \mathbb{Z}(d-1)) \stackrel{\text { def }}{=} \lim _{\rightarrow} H^{d}\left(E, \mu_{n}^{\otimes(d-1)}\right),
$$

where the limit is taken over all $n$ prime to the characteristic exponent of $F$.

Let $M$ be a cycle module over $F, L / F$ a finite field extension, $v$ a discrete valuation of $L$ over $F$. An element $a \in M^{d}(L)$ is called unramified with respect to $v$ if $a$ belongs to the kernel of the residue homomorphism

$$
\partial_{v}: M^{d}(L) \longrightarrow M^{d-1}(F(v)) .
$$

An element $a \in M^{d}(L)$ is unramified over $F$ if it is unramified with respect to all discrete valuations of $L$ over $F$. We denote the subgroup in $M^{d}(L)$ of all unramified over $F$ elements by $M_{\mathrm{nr}}^{d}(L)$ (cf. [5]).

Let $i: E \rightarrow L$ be a field homomorphism over $F, v$ a discrete valuation of $L$ over $F, v^{\prime}$ the restriction of $v$ on $E$. Assume that an element $a \in M^{d}(E)$ is unramified with respect to $v^{\prime}$ (if $v^{\prime}$ is not trivial). By rules R3a and R3c in [13], the element $i_{*}(a) \in M^{d}(L)$ is unramified with respect to $v$. Hence, $i_{*}$ takes $M_{\mathrm{nr}}^{d}(E)$ into $M_{\mathrm{nr}}^{d}(L)$, making $M_{\mathrm{nr}}^{d}$ a functor from Fields $/ F$ to $\mathbf{A b}$.

\section{PROPOSITION 2.3. - The functor $M_{\mathrm{nr}}^{d}$ is homotopy invariant.}

Proof. - Let $L / F$ be a field extension and $i: L \rightarrow L(t)$ the inclusion. By homotopy property $[13,2.2(\mathrm{H})]$, the homomorphism $i_{*}: M^{d}(L) \rightarrow M^{d}(L(t))$ is injective and the image of $i_{*}$ consists of all elements in $M^{d}(L(t))$ that are unramified with respect to all discrete valuation on $L(t)$ over $L$. Therefore, for every $m \in M_{\mathrm{nr}}^{d}(L(t))$ there is (unique) $m^{\prime} \in M^{d}(L)$ such that $i_{*}\left(m^{\prime}\right)=m$, and we need to show that $m^{\prime} \in M_{\mathrm{nr}}^{d}(L)$.

Let $v$ be any discrete valuation of $L$ over $F$ with residue field $F(v)$ and let $v^{\prime}$ be an extension of $v$ to $L(t)$ with ramification index 1 and residue field $F(v)(t)$. Denote by $j$ the inclusion 
$F(v) \rightarrow F(v)(t)$. By rule R3a in [13], the diagram

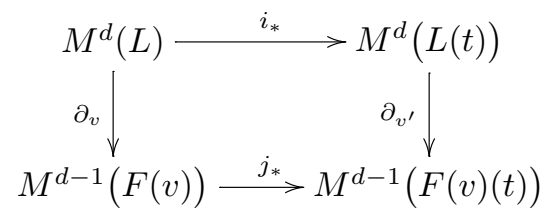

commutes. Since $j_{*}$ is injective and $\partial_{v^{\prime}}(m)=0$, it follows that $\partial_{v}\left(m^{\prime}\right)=0$, i.e., $m^{\prime}$ is unramified.

COROLlARY 2.4. - For any algebraic group $G$, the group $M_{\mathrm{nr}}^{d}(B G)$ is well defined.

Let $X_{\rho}$ be a classifying variety of a group $G$ with respect to an embedding $\rho: G \hookrightarrow S$ with $S$ a special rational group. Consider the group $A^{0}\left(X_{\rho}, M^{d}\right)$ consisting of all elements in $M^{d}\left(F\left(X_{\rho}\right)\right)$ unramified with respect to discrete valuations associated to all irreducible divisors of $X_{\rho}[13, \S 2]$. Thus, we have

$$
M_{\mathrm{nr}}^{d}(B G)=M_{\mathrm{nr}}^{d}\left(F\left(X_{\rho}\right)\right) \subset A^{0}\left(X_{\rho}, M^{d}\right) .
$$

By Corollary A.2, the group $A^{0}\left(X_{\rho}, M^{d}\right)$ does not depend on the choice of $\rho$ if $S$ is a split semisimple simply connected group (for example, $S=\mathbf{S L}_{n}$ or $\mathbf{S p}_{2 n}$ ). We denote by $A^{0}\left(B G, M^{d}\right)$ the group $A^{0}\left(X_{\rho}, M^{d}\right)$ with such a choice of $S$. We have

$$
M_{\mathrm{nr}}^{d}(B G) \subset A^{0}\left(B G, M^{d}\right) .
$$

The unramified group $M_{\mathrm{nr}}^{d}(B G)$ has nice functorial properties with respect to field extensions. Namely, for any field extension $L / F$ there is a well defined restriction homomorphism

$$
\text { res: } M_{\mathrm{nr}}^{d}(B G) \longrightarrow M_{\mathrm{nr}}^{d}\left(B G_{L}\right),
$$

where $B G_{L}=B G \times \times_{\operatorname{Spec} F} \operatorname{Spec} L$. If $L / F$ is finite, the rule R3b in [13] implies the existence of the corestriction homomorphism

$$
\text { cor : } M_{\mathrm{nr}}^{d}\left(B G_{L}\right) \longrightarrow M_{\mathrm{nr}}^{d}(B G) .
$$

Denote by $A^{0}\left(B G, M^{d}\right)_{\text {norm }}$ the kernel of the evaluation (pull-back) homomorphism [13, $\S 12]$

$$
i^{*}: A^{0}\left(B G, M^{d}\right) \longrightarrow A^{0}\left(\operatorname{Spec} F, M^{d}\right)=M^{d}(F)
$$

induced by the distinguished point $i: \operatorname{Spec} F \rightarrow B G$. Thus,

$$
A^{0}\left(B G, M^{d}\right)=M^{d}(F) \oplus A^{0}\left(B G, M^{d}\right)_{\text {norm }} .
$$

Also set

$$
M_{\mathrm{nr}}^{d}(B G)_{\mathrm{norm}}=M_{\mathrm{nr}}^{d}(B G) \cap A^{0}\left(B G, M^{d}\right)_{\mathrm{norm}} .
$$

Thus,

$$
M_{\mathrm{nr}}^{d}(B G)=M^{d}(F) \oplus M_{\mathrm{nr}}^{d}(B G)_{\mathrm{norm}} .
$$

Note that if $B G$ is stably rational, then $M_{\mathrm{nr}}^{d}(B G)_{\text {norm }}=0$ by Proposition 2.3. 


\section{Unramified invariants of algebraic groups}

Let $G$ be an algebraic group defined over a field $F$ and let $M$ be a cycle module over $F$. An invariant of $G$ in $M$ of dimension $d$ is a morphism

$$
H^{1}(*, G) \longrightarrow M^{d}(*)
$$

of functors from the category Fields $/ F$ to the category of sets $[20, \S 6]$. All the invariants of $G$ in $M$ of dimension $d$ form an abelian group $\operatorname{Inv}^{d}(G, M)$.

An element in $M^{d}(F)$ defines a constant invariant of $G$ in $M$. Thus, there is an inclusion

$$
M^{d}(F) \subset \operatorname{Inv}^{d}(G, M) .
$$

An invariant is called normalized if it takes the distinguished element in $H^{1}(F, G)$ to zero (i.e., it can be considered as a morphism of functors with values in the category of pointed sets). We denote the subgroup of normalized invariants by $\operatorname{Inv}^{d}(G, M)_{\text {norm. }}$. Clearly,

$$
\operatorname{Inv}^{d}(G, M)=M^{d}(F) \oplus \operatorname{Inv}^{d}(G, M)_{\text {norm }} .
$$

Let $X=X_{\rho}$ be a classifying variety of $G$ with respect to an embedding of $G$ into a special rational group $S$. An invariant $u \in \operatorname{Inv}^{d}(G, M)$ defines for any field extension $L / F$ the composition

$$
\tilde{u}_{L}: X(L) \longrightarrow H^{1}(L, G) \stackrel{u_{L}}{\longrightarrow} M^{d}(L),
$$

which is constant on orbits of the $S(L)$-action on $X(L)$.

Let $\xi \in X(F(X))$ be the generic point. The image $\tilde{u}_{F(X)}(\xi)$ is an element of the group $M^{d}(F(X))$. A proof of the following Proposition 3.1 and Theorem 3.2 can be found in Appendix A.

PROPOSITION 3.1 (Rost, Serre [18]). - The element $\tilde{u}_{F(X)}(\xi)$ is unramified with respect to the discrete valuation associated to every irreducible divisor of $X$, i.e., $\tilde{u}_{F(X)}(\xi) \in A^{0}\left(X, M^{d}\right)$.

Thus, by Proposition 3.1, we get a homomorphism

$$
\theta: \operatorname{Inv}^{d}(G, M) \longrightarrow A^{0}\left(X, M^{d}\right), \quad u \mapsto \tilde{u}_{F(X)}(\xi) .
$$

THEOREM 3.2 (Rost). - The map $\theta$ is injective. If the special group $S$ is split semisimple simply connected, $\theta$ is an isomorphism.

Thus, for any algebraic group $G$, we have a canonical isomorphism

$$
\theta: \operatorname{Inv}^{d}(G, M) \stackrel{\sim}{\longrightarrow} A^{0}\left(B G, M^{d}\right) .
$$

We say that an invariant $u \in \operatorname{Inv}^{d}(G, M)$ is unramified if $\theta(u) \in M_{\mathrm{nr}}^{d}(B G)$ and normalized if $u(1)=0$. We denote the groups of unramified (respectively normalized) invariants by $\operatorname{Inv}_{\mathrm{nr}}^{d}(G, M)$ (respectively $\left.\operatorname{Inv}^{d}(G, M)_{\text {norm }}\right)$.

LemMA 3.3. - Let $E / F$ be a field extension with $\operatorname{tr} \cdot \operatorname{deg}(E / F) \geqslant \operatorname{dim} X+\operatorname{dim} S$. Then for every point $x \in X(E)$ there is $s \in S(E)$ such that the point $s x \in X(E)$, considered as a morphism $\operatorname{Spec} E \rightarrow X$, is dominant. 
Proof. - Let $Y$ be the closure of the image of $x: \operatorname{Spec} E \rightarrow X$. The function field $F(Y)$ can be considered as a subfield in $E$. Since $\operatorname{tr} \cdot \operatorname{deg}(E / F) \geqslant \operatorname{dim} X+\operatorname{dim} S$, there is a field between $F(Y)$ and $E$, purely transcendental over $F(Y)$ of degree $\operatorname{dim} S$. Since $S$ is rational, we can embed the function field $F(S \times Y)$ into $E$ over $F(Y)$. The composition

$$
f: \operatorname{Spec} E \longrightarrow \operatorname{Spec} F(S \times Y) \longrightarrow S \times Y
$$

is dominant and defines a point $s \in S(E)$. The point $s x$ is given by the composition

$$
s x: \operatorname{Spec} E \stackrel{f}{\longrightarrow} S \times Y \stackrel{m}{\longrightarrow} X,
$$

where $m$ is the restriction of the action morphism. Since $S$ acts transitively on $X, m$ is dominant and therefore so is $s x$.

The following proposition provides a useful tool to determine whether a given invariant is unramified.

Proposition 3.4. - An invariant $u \in \operatorname{Inv}^{d}(G, M)$ is unramified if and only if for any field extension $L / F$ and for every point $y \in H^{1}(L((t)), G)$ the element $u(y) \in M^{d}(L((t)))$ is unramified with respect to the canonical discrete valuation on $L((t))$ over $L$.

Proof. - Assume that $u(y) \in M^{d}(L((t)))$ is unramified for any field extension $L / F$ and every $y \in H^{1}(L((t)), G)$. Let $X$ be a classifying variety of $G, v$ a discrete valuation on $F(X)$ over $F$. The completion $E$ of $F(X)$ with respect to $v$ is isomorphic to $L((t))$, where $L$ is the residue field of $v$. Let $y \in H^{1}(E, G)$ be the image of the generic point $\xi$ under the composition

$$
X(F(X)) \longrightarrow H^{1}(F(X), G) \rightarrow H^{1}(E, G)
$$

induced by the embedding $i: F(X) \hookrightarrow E$. By assumption, the element $u(y)$ is unramified with respect to the extension $v^{\prime}$ on $E$ of the valuation $v$. The composition

$$
M^{d}(F(X)) \stackrel{i_{*}}{\longrightarrow} M^{d}(E) \stackrel{\partial_{v^{\prime}}}{\longrightarrow} M^{d-1}(L)
$$

coincides with $\partial_{v}$. Hence,

$$
\partial_{v}(u(\xi))=\partial_{v^{\prime}}\left(i_{*}(u(\xi))\right)=\partial_{v^{\prime}}(u(y))=0,
$$

i.e., $u$ is unramified.

Conversely, assume that $u$ is unramified. Let $L / F$ be a field extension and $y \in H^{1}(L((t)), G)$. Choose a point $x \in X(L((t)))$ representing $y$. By Lemma 3.3, we may assume that the point $x$, considered as a morphism $\operatorname{Spec} L((t)) \rightarrow X$, is dominant. Thus, the function field $F(X)$ is isomorphic to a subfield in $L((t))$. The natural homomorphism induced by the field extension $L((t)) / F(X)$,

$$
X(F(X)) \longrightarrow X(L((t)))
$$

takes the generic point $\xi$ to $x$, hence the map

$$
M^{d}(F(X)) \longrightarrow M^{d}(L((t)))
$$

takes $u(\xi)$ to $u(y)$. Since $u(\xi)$ is unramified, so is $u(y)$. 


\section{Rost invariants}

We will be considering the following cohomological cycle module $H$ over $F$ (Example 2.2):

$$
H^{d}(L)=H^{d}(L, \mathbb{Q} / \mathbb{Z}(d-1))
$$

for a field extension $L / F$. We shall compute the unramified groups

$$
H_{\mathrm{nr}}^{3}(B G) \simeq \operatorname{Inv}_{\mathrm{nr}}^{3}(G, H)
$$

for every (connected) semisimple simply connected group $G$. The following propositions reduce the problem to the case of an absolutely simple simply connected group $G$. By Corollary B.3, in this case the group $\operatorname{Inv}^{3}(G, H)_{\text {norm }}$ is finite cyclic with a canonical generator $r_{G}$ (Rost invariant). In the following sections we consider all absolutely simple groups of classical types $A_{n}, B_{n}, C_{n}$ and $D_{n}$.

An arbitrary simply connected group $G$ is a product of simple simply connected groups $G_{1} \times G_{2} \times \cdots \times G_{k}[21,3.1 .2]$. The functorial properties of $H_{\mathrm{nr}}^{3}$ considered in (2.2) and Corollary B.4 imply

PROPOSITION 4.1. - $H_{\mathrm{nr}}^{3}(B G)_{\mathrm{norm}} \simeq \coprod_{i=1}^{k} H_{\mathrm{nr}}^{3}\left(B G_{i}\right)_{\mathrm{norm}}$.

Any simple simply connected group $G$ is of the form $R_{L / F}\left(G^{\prime}\right)$, where $L / F$ is a finite separable field extension and $G^{\prime}$ is an absolutely simple simply connected group over $L$ [21, 3.1.2]. By Corollary B.5, the two compositions $j^{*} \circ \operatorname{res}_{L / F}$ and $\operatorname{cor}_{L / F} \circ i^{*}$ in the diagram

$$
H_{\mathrm{nr}}^{3}(B G)_{\mathrm{norm}} \underset{\operatorname{cor}_{L / F}}{\stackrel{\mathrm{res}_{L / F}}{\rightleftarrows}} H_{\mathrm{nr}}^{3}\left(B G_{L}\right)_{\text {norm }} \underset{i^{*}}{\stackrel{j^{*}}{\gtrless}} H_{\mathrm{nr}}^{3}\left(B G^{\prime}\right)_{\text {norm }}
$$

are isomorphisms inverse to each other.

PROPOSITION 4.2. $-H_{\mathrm{nr}}^{3}(B G)_{\mathrm{norm}} \simeq H_{\mathrm{nr}}^{3}\left(B G^{\prime}\right)_{\text {norm }}$.

We will need the following lemmas. The definition and properties of the numbers $n_{\alpha}, n_{G}, n_{G}^{\prime}$ and the Rost invariant $r_{G}$ are collected in Appendix B.

LEMMA 4.3. - Let $\alpha: H \rightarrow G$ be a homomorphism of absolutely simple simply connected groups with $n_{\alpha}=1$. If $H_{\mathrm{nr}}^{3}(B H)_{\mathrm{norm}}=0$ and $n_{H}^{\prime}=n_{G}^{\prime}$, then $H_{\mathrm{nr}}^{3}(B G)_{\mathrm{norm}}=0$.

Proof. - The image of $r_{G}$ in $A^{0}(B H, H)_{\text {norm }}$ is equal to $r_{H}$ since $n_{\alpha}=1$. Assume that $m r_{G}$ is unramified for some $m \in \mathbb{Z}$. It follows from $H_{\mathrm{nr}}^{3}(B H)_{\text {norm }}=0$ that $m r_{H}=0$ and hence $m$ is divisible by $n_{H}^{\prime}=n_{G}^{\prime}$. Therefore, $m r_{G}=0$.

LEMMA 4.4. - Let $G$ be an absolutely simple simply connected group over $F$. Assume that for a field extension $L / F, n_{G_{L}}^{\prime}=n_{G}^{\prime}$ and $H_{\mathrm{nr}}^{3}\left(B G_{L}\right)_{\mathrm{norm}}=0$. Then $H_{\mathrm{nr}}^{3}(B G)_{\mathrm{norm}}=0$.

Proof. - Assume that $m r_{G}$ is unramified for some $m \in \mathbb{Z}$. Since over $L$ this element becomes trivial, $m$ is divisible by $n_{G_{L}}^{\prime}=n_{G}^{\prime}$. Hence, $m r_{G}=0$.

In the following sections we compute the groups $H_{\mathrm{nr}}^{3}(B G)_{\text {norm }}$ for all absolutely simple simply connected algebraic groups $G$ of classical types. We follow the classification of simple groups given in $[9, \mathrm{Ch} .6]$. 


\section{Type $A_{n-1}$}

\subsection{Inner type}

Let $G$ be a simply connected group of inner type $A_{n-1}$, i.e., $G=\mathbf{S L}_{1}(A)$ for a central simple $F$-algebra $A$ of degree $n$. We embed $G$ into the special rational group $\mathbf{G L}_{1}(A)$ with the classifying variety $X=\mathbb{G}_{m}$. Since $X$ is rational, $M_{\mathrm{nr}}^{d}(B G)_{\text {norm }}=0$ for any cycle module $M$ over $F$.

THEOREM 5.1. - Let $G$ be a simply connected group of inner type $A_{n}$. Then a classifying variety $B G$ is stably rational and $M_{\mathrm{nr}}^{d}(B G)_{\mathrm{norm}}=0$ for any cycle module $M$ over $F$.

\subsection{Outer type}

Let $G$ be a simply connected group of outer type $A_{n-1}$, i.e., $G=\mathbf{S U}(B, \tau)$, where $B$ is a central simple algebra of degree $n \geqslant 3$ with unitary involution $\tau$ over a quadratic separable field extension $K / F$.

Over $K$, the group $G_{K}$ is isomorphic to $\mathbf{S L}_{1}(B)$ and by B.3.1, a value of the Rost invariant $r_{G_{K}}$ over a field extension $L / K$ is of the form $(x) \cup\left[B_{L}\right]$ for some $x \in L^{\times}$. Hence, taking the norm of the invariant $r_{G_{K}}$ in the extension $K / F$, we conclude that a value of the invariant $2 r_{G}$ over a field extension $E / F$ is of the form

$$
N_{K \otimes E / E}\left((x) \cup\left[B_{K \otimes E}\right]\right) \in H^{3}(E, \mathbb{Q} / \mathbb{Z}(2))
$$

for some $x \in(K \otimes E)^{\times}$.

LEMMA 5.2. - If $\exp (B)$ is even, the invariant $\exp (B) r_{G}$ is unramified.

Proof. - Let $L / F$ be a field extension. By Proposition 3.4, it suffices to show that for every $y \in H^{1}(L((t)), G)$ the element $\exp (B) r_{G}(y)$ in $H^{3}(L((t)), \mathbb{Q} / \mathbb{Z}(2))$ is unramified with respect to the canonical valuation $v$ of $L((t))$. Consider two cases.

Case 1: $K \otimes L$ is not a field, i.e., the group $G_{L((t))}$ is of inner type. By (B.3.1), the order of the Rost invariant over $L((t))$ is equal to $\exp \left(B_{L((t))}\right)^{\prime}$, hence $\exp (B) r_{G}$ is zero over $L((t))$ and obviously $\exp (B) r_{G}(y)=0$ is unramified.

Case 2: $K L=K \otimes L$ is a field. Since $\exp (B)$ is even, it suffices to show that $\partial_{v}\left(2 r_{G}(y)\right)=0$. We have by (1),

$$
2 r_{G}(y)=N_{K L((t)) / L((t))}\left((x) \cup\left[B_{K L((t))}\right]\right)
$$

for some $x \in K L((t))^{\times}$. Then for the valuation $v^{\prime}$ of $K L((t))$,

$$
\partial_{v}\left(2 r_{G}(y)\right)=\partial_{v}\left(N_{K L((t)) / L((t))}\left((x) \cup\left[B_{K L((t))}\right]\right)\right)=N_{K L / L}\left[B_{K L}\right]^{v^{\prime}(x)}=0
$$

since $B_{K L}$ has unitary involution $\tau_{K L}$ and therefore

$$
N_{K L / L}\left[B_{K L}\right]=0 \in H^{2}(L, \mathbb{Q} / \mathbb{Z}(1))
$$

by $[9$, Th. 3.1].

Denote by $D=D(B, \tau)$ the discriminant algebra of $(B, \tau)[9, \S 10]$.

THEOREM 5.3. - Assume that $\operatorname{char}(F) \neq 2$. Let $G=\mathbf{S U}(B, \tau)$, where $B$ is a central simple algebra of degree $n \geqslant 3$ with unitary involution $\tau$ over a quadratic field extension $K / F$. Then 
the group $H_{\mathrm{nr}}^{3}(B G)_{\mathrm{norm}}$ is cyclic of order 2 generated by $\exp (B) r_{G}$, except for the following cases (when this group is trivial):

(1) $\exp (B)$ is odd;

(2) $n$ is a 2-power and $\exp (B)=n$;

(3) $n$ is a 2-power, $\exp (B)=n / 2$ and the discriminant algebra $D$ is split.

Proof. - Assume that the invariant $m r_{G}$ is unramified for some $m \in \mathbb{Z}$. Since over $K$, the group $H_{\mathrm{nr}}^{3}\left(B G_{K}\right)_{\text {norm }}$ is trivial by Theorem 5.1, the class $m r_{G}$ vanishes over $K$. It is shown in B.3.1 that over $K, r_{G}$ has order $\exp (B)^{\prime}$, therefore $\exp (B)^{\prime} \mid m$. Thus, since $\operatorname{char}(F) \neq 2$ and $\exp (B)\left|n_{G}\right| 2 \exp (B)$ by Theorem B.20, the group $H_{\mathrm{nr}}^{3}(B G)_{\text {norm }}$ consists of at most two elements and it is cyclic of order 2 if and only if $n_{G}=2 \exp (B)$ and the invariant $\exp (B) r_{G}$ is unramified.

Assume first that $B$ splits, i.e., $B=\operatorname{End}_{K}(V)$, where $V$ is a vector space over $K$ of dimension $n \geqslant 3$. The involution $\tau$ is adjoint with respect to a hermitian form $h$ on $V$ over $K / F$ [9, Th. 4.2]. Theorem B.20 gives $n_{G}=2$ over any field extension of $F$. By Lemma 4.4, it is sufficient to prove that $H_{\mathrm{nr}}^{3}\left(B G_{L}\right)_{\text {norm }}=0$ for some field extension $L / F$.

We claim that over a field extension $E / F$, there is a non-degenerate subform $\left(V_{0}, h_{0}\right)$ in $\left(V \otimes_{F} E, h \otimes_{F} E\right)$ of dimension 2 and non-trivial discriminant disc $\left(h_{0}\right)$. To prove the claim we consider two cases. If $h$ is anisotropic, we can take $E=F$ and $h_{0}$ an arbitrary subform of $h$ of dimension 2. Assume that $h$ is isotropic, $h=h^{\prime} \perp \mathbb{H}$, where $\mathbb{H}$ is the hyperbolic plane. Let $a \in F^{\times}$be a value of $h^{\prime}$. The form $\mathbb{H}$ is universal, hence the form $h_{0}=\langle a, t\rangle$ is a subform of $h$ over $E=F(t)$. The discriminant $\operatorname{disc}\left(h_{0}\right)$ is not trivial as at is not a norm in the quadratic extension $K(t) / F(t)$.

Now we can replace $F$ by $E$ and consider the subgroup $H=\mathbf{S U}\left(V_{0}, h_{0}\right) \subset G$. The group $H$ is a simply connected group of (inner) type $A_{1}$, therefore, $H=\mathbf{S L}_{1}(Q)$ for a quaternion algebra $Q$ [9, Th. 26.9]. Since the discriminant of $h_{0}$ is not trivial, $H$ is not split and hence $Q$ does not split. Thus, $n_{H}=2$ by Theorem B.17. Let $\rho: H \hookrightarrow G$ be the embedding. By Example B.6, $n_{\rho}=1$. Hence, the inner case 5.1 and Lemma 4.3, applied to the embedding $\rho$, imply that $H_{\mathrm{nr}}^{3}(B G)_{\text {norm }}=0$.

Now assume that $\exp (B)$ is odd. We have $n_{G}=2 \exp (B)$ by Theorem B.20. The first part of the proof shows that the non-trivial invariant $\exp (B) r_{G}$ ramifies over any field extension of $F$ which splits $B$ but not $K$ (for example, the function field of the variety $R_{K / F}(\mathrm{SB}(B)$ ), where $\mathrm{SB}(B)$ is the Severi-Brauer variety of $B[9, \S 1 . C])$. Hence, $\exp (B) r_{G}$ already ramifies over $F$ and therefore the group $H_{\mathrm{nr}}^{3}(B G)_{\text {norm }}$ is trivial.

Finally, assume that $\exp (B)$ is even. By Lemma $5.2, \exp (B) r_{G}$ is unramified, i.e., the group $H_{\mathrm{nr}}^{3}(B G)_{\text {norm }}$ is cyclic of order 2 if and only if $n_{G}=2 \exp (B)$. The result follows from Theorem B.20.

Corollary 5.4. - Assume that $\operatorname{char}(F) \neq 2$. Let $G=\mathbf{S U}(B, \tau)$ with $\exp (B)$ even and $\operatorname{deg}(B) \geqslant 4$. Assume in addition that if $\operatorname{deg}(B)=4$, the discriminant algebra $D(B, \tau)$ does not split. Then a classifying variety $B G$ is not stably rational.

Proof. - Consider the field extension $L=F\left(R_{K / F}\left(\mathrm{SB}\left(B^{\otimes 2}\right)\right)\right)$. By the index reduction formula [17, §3], $D(B, \tau)$ is not split over $L$ and $\exp \left(B_{L}\right)=2$, so that, extending the base field to $L$, we may assume that $\exp (B)=2$. Then, by Theorem 5.3, the unramified group $H_{\mathrm{nr}}^{3}(B G)_{\text {norm }}$ is not trivial.

Remark 5.5. - Examples of stably non-rational classifying varieties $B G$ with simply connected $G$ of type $A_{n}$ exist for every odd $n \geqslant 3$. Every number field can be taken for the base field $F$. 


\section{Type $B_{n}$}

Let $G$ be a simply connected group of type $B_{n}, n \geqslant 2$, i.e., $G=\operatorname{Spin}(V, q)$, where $(V, q)$ is a non-degenerate quadratic form of dimension $2 n+1$.

If $n=2$, we have $B_{2}=C_{2}$ and $H_{\mathrm{nr}}^{3}(B G)_{\text {norm }}=0$ by Theorem 7.1.

Assume that $n \geqslant 3$. We claim that over some field extension of $F,(V, q)$ contains a nondegenerate subform $\left(V^{\prime}, q^{\prime}\right)$ of dimension 5 and of Witt index at most 1 . To prove the claim, we may assume first that $q$ is hyperbolic. Let $f$ be anisotropic 3-dimensional form over some field extension $L / F$. Since $\operatorname{dim} q \geqslant 7, f$ is isomorphic to a subform of $q_{L}$. The Witt index of any 5-dimensional form $q^{\prime}$ such that $f \subset q^{\prime} \subset q_{L}$ is at most 1 . The claim is proved.

The group $H=\operatorname{Spin}\left(V^{\prime}, q^{\prime}\right)$ is a subgroup of $G_{L}$ of type $B_{2}=C_{2}$. We have $n_{H}=n_{G}=$ $n_{G_{L}}=2$ by Theorem B.22 and $H_{\mathrm{nr}}^{3}(B H)_{\text {norm }}=0$ by the case $n=2$. Example B.10 shows that $n_{\rho}=1$ for the embedding $\rho: H \hookrightarrow G$. Lemma 4.3 implies that $H_{\mathrm{nr}}^{3}\left(B G_{L}\right)_{\text {norm }}=0$ and by Lemma 4.4, $H_{\mathrm{nr}}^{3}(B G)_{\text {norm }}=0$.

THEOREM 6.1. - Let $G$ be a simply connected group of type $B_{n}$. Then $H_{\mathrm{nr}}^{3}(B G)_{\mathrm{norm}}=0$.

\section{Type $C_{n}$}

Let $G$ be a simply connected group of type $C_{n}, n \geqslant 2$, i.e., $G=\mathbf{S p}(A, \sigma)$, where $A$ is a central simple $F$-algebra of degree $2 n$ with a symplectic involution $\sigma$. We consider the canonical embedding

$$
\rho: \mathbf{S p}(A, \sigma) \smile \mathbf{S L}_{1}(A)
$$

The map $x \mapsto \sigma(x) x$ establishes an isomorphism between the classifying space $X_{\rho}$ and the open subvariety in the linear space

$$
\operatorname{Symd}(A, \sigma)=\{a+\sigma(a), a \in A\}
$$

consisting of all invertible elements [9, 29.24]. This variety is rational (being an open subset of an affine space), hence $M_{\mathrm{nr}}^{d}(B G)_{\text {norm }}=0$ for any cycle module $M$ over $F$.

THEOREM 7.1. - Let $G$ be a simply connected group of type $C_{n}$. Then a classifying variety $B G$ is stably rational and $M_{\mathrm{nr}}^{d}(B G)_{\mathrm{norm}}=0$ for any cycle module $M$ over $F$.

\section{Type $D_{n}$}

We assume char $F \neq 2$. Let $G$ be a simply connected group of type $D_{n}$ (we exclude groups of trialitarian type in $\left.D_{4}\right)$, i.e., $G=\operatorname{Spin}(A, \sigma)$ for a central simple algebra $A$ of degree $2 n$ over $F$ with an orthogonal involution $\sigma$. The standard isogeny

$$
\alpha: G \longrightarrow \mathbf{O}^{+}(A, \sigma)
$$

induces a map

$$
\alpha_{*}: H^{1}(F, G) \longrightarrow H^{1}\left(F, \mathbf{O}^{+}(A, \sigma)\right) .
$$

Let $X$ be the variety of pairs

$$
(a, x) \in \operatorname{Sym}(A, \sigma) \times F^{\times}
$$


such that $\operatorname{Nrd}(a)=x^{2}$. The morphism

$$
\mathbf{G L}_{1}(A) \longrightarrow X, \quad g \mapsto(g \sigma(g), \operatorname{Nrd}(g))
$$

induces an isomorphism of varieties $\mathbf{G L}_{1}(A) / \mathbf{O}^{+}(A, \sigma) \stackrel{\sim}{\rightarrow} X$ making $X$ a classifying variety of $\mathbf{O}^{+}(A, \sigma)$ and identifying the set $H^{1}\left(F, \mathbf{O}^{+}(A, \sigma)\right)$ with the factor set of $X(F)$ modulo the action of the group $\mathrm{GL}_{1}(A)$ given by $g(a, x)=(\operatorname{ga\sigma }(g), \operatorname{Nrd}(g) x)[9,29.27]$.

The embedding

$$
\beta: \mathbf{O}^{+}(A, \sigma) \longleftrightarrow \mathbf{S L}_{1}(A)
$$

induces the morphism $X \rightarrow \mathbb{G}_{m}=\mathbf{G L}_{1}(A) / \mathbf{S L}_{1}(A)$ taking a pair $(a, x)$ to $x$. Thus, the map

$$
\beta_{*}: H^{1}\left(F, \mathbf{O}^{+}(A, \sigma)\right) \longrightarrow H^{1}\left(F, \mathbf{S L}_{1}(A)\right)=F^{\times} / \operatorname{Nrd}\left(A^{\times}\right)
$$

takes the class represented by a pair $(a, x)$ to $x \operatorname{Nrd}\left(A^{\times}\right)$.

By Example B.11, $n_{\beta \circ \alpha}=2$, hence the Rost invariant for $\mathbf{S L}_{1}(A)$ corresponds to $2 r_{G}$ under $\beta \circ \alpha$ and therefore, by B.3.1, for any field extension $L / F$ and every $y \in H^{1}(L, G)$,

$$
2 r_{G}(y)=(x) \cup\left[A_{L}\right] \in H^{3}(L, \mathbb{Q} / \mathbb{Z}(2)),
$$

provided the class $\alpha_{*}(y)$ is represented by a pair $(a, x) \in X(F)$.

Let $Q$ be a quaternion division algebra and let $(V, h)$ be a $(-1)$-hermitian forms over $Q$ with respect to the canonical (symplectic) involution on $Q$. Assume that discriminant of $h$ (i.e., discriminant of the adjoint involution $\sigma_{h}$ on $\operatorname{End}_{Q}(V)$ ) is trivial. Then the Clifford algebra $C\left(\operatorname{End}_{Q}(V), \sigma_{h}\right)$ is a product of two central simple $F$-algebras $C^{+}(h)$ and $C^{-}(h)[9, \S 8]$. If in addition $\operatorname{dim}_{Q} V$ is even, exponent of the algebras $C^{ \pm}$is at most 2 [9, Th. 9.13].

LEMMA 8.1. - Let $k$ and $l$ be two $(-1)$-hermitian forms over $Q$ with respect to the canonical involution on $Q$. Assume that $\operatorname{rank}(k)+\operatorname{rank}(l)$ is even and the discriminant of the form $k_{F((t))} \perp t l_{F((t))}$ over $F((t))$ is trivial. Then

$$
\partial_{v}\left[C^{ \pm}\left(k_{F((t))} \perp t l_{F((t)))}\right]=\operatorname{disc}(l) \in F^{\times} / F^{\times^{2}},\right.
$$

where $v$ is the discrete valuation on $F((t))$ and $\partial_{v}:{ }_{2} \operatorname{Br} F((t)) \rightarrow F^{\times} / F^{\times^{2}}$ is the residue homomorphism.

Proof. - We can split $Q$ generically (by the function field of the conic curve corresponding to $Q$ ) and assume that we are given two quadratic forms $f$ and $g$ of even dimension such that the form $f_{F((t))} \perp t g_{F((t))}$ has trivial discriminant. Denote by $I F$ the fundamental ideal in the Witt ring of $F[10]$. The commutativity of the diagram

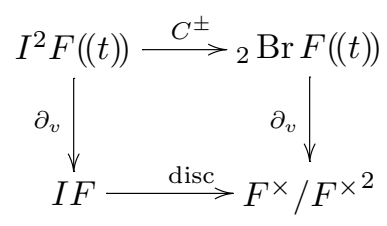

and description of the residue homomorphisms in [10] yield the result.

Proposition 8.2. - If $n=4$ and $\operatorname{disc}(\sigma)$ is trivial, then the invariant $2 r_{G}$ is unramified. 
Proof. - By Proposition 3.4, it suffices to prove that for any field extension $L / F$ and every $y \in H^{1}(L((t)), G)$, the residue $\partial_{v}\left(2 r_{G}(y)\right)$ is trivial. We may assume that $L=F$.

We have by (2),

$$
2 r_{G}(y)=(x) \cup\left[A_{F((t))}\right] \in H^{3}(F((t)), \mathbb{Q} / \mathbb{Z}(2))
$$

with $x \in F((t))^{\times}$such that $x^{2}=\operatorname{Nrd}(a)$ for some $a \in \operatorname{Sym}\left(A_{F((t))}, \sigma_{F((t))}\right)$. Hence

$$
\partial_{v}\left(2 r_{G}(y)\right)=[A]^{v(x)} \in H^{2}(F, \mathbb{Q} / \mathbb{Z}(1))=\operatorname{Br} F .
$$

Thus, we may assume that $A$ is not split. Since then $\exp (A)=2$, it suffices to prove that $v(x)$ is even. Assume that $v(x)$ is odd. The integer $v(\operatorname{Nrd} a)$ is divisible by ind $(A), v(x)=v(\operatorname{Nrd} a) / 2$ is divisible by $\operatorname{ind}(A) / 2$. Therefore, $\operatorname{ind}(A)=2$, i.e., $A$ is similar to a quaternion division algebra $Q$ over $F, A \simeq M_{4}(Q)$.

By [9, Th. 4.2], the involution $\sigma$ is adjoint to a (-1)-hermitian form $h$ of rank 4 over $Q$ with respect to the canonical involution on $Q$. The symmetric element $a$ gives rise to another $(-1)$-hermitian form $h^{\prime}$ of rank 4 over $Q_{F((t))}$ with trivial discriminant. We diagonalize this form by choosing an element $g \in \mathrm{GL}_{1}\left(A_{F((t))}\right)$ such $g a \sigma(g)$ is the diagonal matrix $\operatorname{diag}\left(t^{e_{1}} q_{1}, t^{e_{2}} q_{2}, t^{e_{3}} q_{3}, t^{e_{4}} q_{4}\right)$, where $q_{i} \in Q^{\times}$are pure quaternions and $e_{i}=0$ or 1 . We have

$$
t^{2 \sum e_{i}} \cdot \prod \operatorname{Nrd}\left(q_{i}\right)=\prod \operatorname{Nrd}\left(t^{e_{i}} q_{i}\right)=\operatorname{Nrd}(g)^{2} \operatorname{Nrd}(a)=\operatorname{Nrd}(g)^{2} x^{2} .
$$

Since $v(\operatorname{Nrd} g)$ is divisible by ind $(A)$ and hence even and $v(x)$ is odd, the sum of the $e_{i}$ is odd. There are two cases:

Case 1: $\sum e_{i}=1$. We may assume that $e_{1}=1$ and $e_{2}=e_{3}=e_{4}=0$. The pair $(a, x)$ belongs to the image of

$$
H^{1}(F((t)), G) \longrightarrow H^{1}\left(F((t)), \mathbf{O}^{+}(A, \sigma)\right) .
$$

By [8], one of the components $C^{+}$and $C^{-}$of the Clifford algebra of the form $h_{F((t))} \perp-h^{\prime}$ splits. By Lemma 8.1, disc $\left\langle q_{1}\right\rangle$ is trivial, i.e., $-\operatorname{Nrd} q_{1}=y^{2}$ for some $y \in F^{\times}[9,7.2]$. Hence $\operatorname{Nrd}\left(y+q_{1}\right)=0$, a contradiction, since $Q$ is a division algebra.

Case 2: $\sum e_{i}=3$. We may assume that $e_{1}=e_{2}=e_{3}=1$ and $e_{4}=0$. As in case 1 , by Lemma 8.1, $\operatorname{disc}\left\langle q_{1}, q_{2}, q_{3}\right\rangle$ is trivial, i.e., $-\operatorname{Nrd}\left(q_{1} q_{2} q_{3}\right)$ is a square in $F^{\times}$. Since the form $h^{\prime}$ has trivial discriminant $\operatorname{disc}\left(h^{\prime}\right)=\operatorname{Nrd}\left(q_{1} q_{2} q_{3} q_{4}\right)$, it follows that $-\operatorname{Nrd} q_{4}$ is also a square in $F^{\times}$, a contradiction as in case 1 .

LEMMA 8.3. - Assume $A$ is not split, $n \geqslant 4$ and in the case $n=4$ the discriminant of $\sigma$ is not trivial. Then there is a field extension $L / F$ and an element $y \in H^{1}(L((t)), G)$ such that $2 r_{G}(y)$ ramifies.

Proof. - Denote by $S$ the generalized Severi-Brauer variety $\operatorname{SB}(2, A)[9,1.16]$. Replacing $F$ by $F(S)$, we can get $A$ similar to a quaternion division algebra $Q=(a, b), A \simeq M_{n}(Q)$ by [1]. Let $W$ be the quadric hypersurface given by the quadratic form $\langle 1,1,-a,-b, a b\rangle$. The field $F(W)$ does not split $Q$ by [10, Ch. IX]. Thus, we may replace $F$ by $F(W)$ and therefore assume that there is an element $q \in Q$ with $\operatorname{Nrd}(q)=-1$. Every element of $Q$ is a product of two pure quaternions. Hence there are pure quaternions $q_{1}, q_{2}$ and $q_{3}$ such that $q_{1} q_{2} q_{3}=q$.

The involution $\sigma$ is adjoint to a $(-1)$-hermitian form $h$ of rank $n$ over $Q$. We claim that there is a $(-1)$-hermitian form $h^{\prime \prime}$ of rank $n-3$ over $Q$ (maybe over some field extension of $F$ which does not split $Q$ ) such that discriminants of the $(-1)$-hermitian forms

$$
h^{\prime}=h_{F((t))}^{\prime \prime} \perp t\left\langle q_{1}, q_{2}, q_{3}\right\rangle
$$


and $h_{F((t))}$ over $F((t))$ coincide, i.e., $\operatorname{disc}\left(h^{\prime \prime}\right)=\operatorname{disc}(h)$. Consider two cases.

Case 1: $n \geqslant 5$. The determinant of a $(-1)$-hermitian form is the product of reduced norms of pure quaternions of a diagonalization. Every element of $Q$ is a product of two pure quaternions, hence every value of the reduced norm of $Q$ can be the determinant of a (-1)-hermitian form of rank at least 2 . This is the case with $h^{\prime \prime}$, $\operatorname{since} \operatorname{rank}\left(h^{\prime \prime}\right)=n-3 \geqslant 2$.

Case 2: $n=4$. Let $i, j$ be the generators of $Q, i^{2}=a, j^{2}=b, i j=-j i$. Consider projective quadric hypersurface given by the equation

$$
a X^{2}+b Y^{2}-a b Z^{2}=c T^{2}
$$

where $c \in F^{\times}$represents $\operatorname{disc}(h)=\operatorname{disc}(\sigma)$. Let $L$ be the function field of the quadric. Since $c$ is not a square in $F^{\times}$, the field $L$ does not split $Q$ by [10, Ch. IX]. Now we can set $h^{\prime \prime}=\left\langle q^{\prime \prime}\right\rangle$, where $q^{\prime \prime}=X i+Y j+Z i j \in Q_{L}$. Then

$$
\operatorname{disc}\left(h^{\prime \prime}\right)=-\operatorname{Nrd}\left(q^{\prime \prime}\right)=c L^{\times^{2}}=\operatorname{disc}\left(h_{L}\right) .
$$

We replace $F$ by $L$. The claim is proved.

Thus, the hermitian form $h^{\prime}$ represents an element in $H^{1}(F((t)), \mathbf{O}(A, \sigma))$, i.e., a pair $(a, x) \in \operatorname{Sym}\left(A_{F((t))}, \sigma_{F((t))}\right)$ such that $\operatorname{Nrd}(a)=x^{2}$. Let $H$ (respectively $H^{\prime}$ ) be the matrix of $h$ (respectively $\left.h^{\prime}\right)$. By [8], $\operatorname{Nrd}(a)=\operatorname{Nrd}(H) \operatorname{Nrd}\left(H^{\prime}\right)^{-1}$, hence

$$
v(\operatorname{Nrd} a)=v(\operatorname{Nrd} H)-v\left(\operatorname{Nrd} H^{\prime}\right)=0-6=-6 .
$$

Therefore $v( \pm x)=-3$ and the class $( \pm x) \cup\left[A_{F((t))}\right]$ is ramified since

$$
\partial_{v}\left(( \pm x) \cup\left[A_{F((t))}\right]\right)=[A]^{v( \pm x)}=[A] \neq 1 .
$$

It suffices to lift $(a, x)$ or $(a,-x)$ to an element $y$ in the set $H^{1}(F((t)), G)$ (maybe over an extension of $F$ which does not split $A$ ). By [8], we need to split one of the components $C^{+}$and $C^{-}$of the Clifford algebra of the form $h_{F((t))} \perp-h^{\prime}$. It follows from Lemma 8.1 that

$$
\partial_{v}\left[C^{ \pm}\right]=\operatorname{disc}\left\langle q_{1}, q_{2}, q_{3}\right\rangle=-\operatorname{Nrd}\left(q_{1}, q_{2}, q_{3}\right)=-\operatorname{Nrd}(q)=1,
$$

i.e., $C^{+}$and $C^{-}$are defined over $F$ : there are central simple algebras $D^{+}$and $D^{-}$over $F$ such that $\left[C^{ \pm}\right]=\left[D_{F((t))}^{ \pm}\right]$. If both the $D^{ \pm}$are not split over $F$, then each of them is not similar to $A$ since $C^{+}$is similar to $C^{-} \otimes A$ by [9, Th. 9.12]. Hence, the function field of the Severi-Brauer variety of $D^{+}$splits $D^{+}$and also $C^{+}$but does not split $A$.

Now Proposition 3.4 yields

Corollary 8.4. - Assume $A$ is not split, $n \geqslant 4$ and in the case $n=4$ that the discriminant of $\sigma$ is not trivial. Then the invariant $2 r_{G}(x)$ ramifies.

THEOREM 8.5. - Let $(A, \sigma)$ be a central simple algebra over a field $F(\operatorname{char} F \neq 2)$ of degree $2 n \geqslant 6$ with an orthogonal involution $\sigma, C=C(A, \sigma)$ the Clifford algebra, $G=\operatorname{Spin}(A, \sigma)$. Then $H_{\mathrm{nr}}^{3}(B G)_{\mathrm{norm}}$ is trivial, except for the following cases (when this group is cyclic of order 2 generated by $\left.2 r_{G}\right)$ :

(1) $n=3$, $\operatorname{disc}(\sigma)$ is not trivial, $A$ is not split and $\exp (C)=2$;

(2) $n=4, \operatorname{disc}(\sigma)$ is trivial, $A$ is not split and neither component $C^{+}$nor $C^{-}$of $C$ splits. 
Proof. - The case $n=3$ follows from Theorem 5.3 since $D_{3}=A_{3}$. Under this equality, the Clifford algebra $C$ coincides with the algebra $B$ considered in Section 5.1 and the algebra $A$ coincides with the discriminant algebra $D[9, \S 26]$.

Consider the case $n \geqslant 4$. Assume $A$ splits, i.e., $G=\operatorname{Spin}(V, q)$ for a non-degenerate quadratic form $(V, q)$ of dimension $2 n$.

Suppose first that $n=4$. Since $n_{G}=2$ by Theorem B.26, it suffices to show that the invariant $r_{G}$ ramifies. Extending $F$, we assume that $q$ is hyperbolic. The image of the map

$$
H^{1}(L, G) \rightarrow H^{1}\left(L, \mathbf{O}^{+}(V, q)\right)
$$

for a field extension $L / F$ is identified with the set of isomorphism classes of quadratic forms over $L$ of dimension 8 with trivial discriminant and trivial Clifford invariant [9, 31.41], i.e., with the set of isomorphism classes of forms similar to 3 -fold Pfister forms $\langle\langle a, b, c\rangle\rangle$ over $L$. The Rost invariant $r_{G}$ associates to this form its Arason invariant [9, 31.42]

$$
(a) \cup(b) \cup(c) \in H^{3}(L, \mathbb{Q} / \mathbb{Z}(2)) .
$$

Let $L / F$ be a field extension having a nonsplit quaternion algebra $Q=(a, b)$. Consider the form $q=\langle\langle t, a, b\rangle\rangle$ over $L((t))$. It follows from

$$
\partial_{v}\left(r_{G}(q)\right)=\partial_{v}((t) \cup(a) \cup(b))=[Q] \neq 1,
$$

that $r_{G}(q)$ ramifies. By Proposition 3.4, the Rost invariant $r_{G}$ ramifies.

For arbitrary $n \geqslant 4$ we can find a non-degenerate subform $\left(V_{0}, q_{0}\right)$ in $(V, q)$ of dimension 8 . Then $H=\operatorname{Spin}\left(V_{0}, q_{0}\right)$ is a subgroup in $G=\operatorname{Spin}(V, q)$. By Theorem B.27, $n_{H}=n_{G}=2$. It follows from the case $n=4$ of the proof, Example B.10 and Lemma 4.3 that $H_{\mathrm{nr}}^{3}(B G)_{\text {norm }}=0$.

Now assume that $A$ is not split. By Theorems B.26 and B.27, $n_{G}$ divides 4 . Let $L$ be any splitting field for $A$. As shown above, the Rost invariant $r_{G}$ ramifies over $L$ and hence ramifies over $F$. Hence the group $H_{\mathrm{nr}}^{3}(B G)_{\text {norm }}$ is nontrivial if and only if the invariant $2 r_{G}$ is nontrivial and unramified. Now the statement follows from Proposition 8.2, Corollary 8.4 and Theorems B.26 and B.27.

COROLlary 8.6. - A classifying variety $B G$ for the group $G=\operatorname{Spin}(A, \sigma)$ is not stably rational in the following cases:

(1) $n=3, \operatorname{disc}(\sigma)$ is not trivial and the algebras $A$ and $C$ are not split;

(2) $n=4, \operatorname{disc}(\sigma)$ is not trivial and the algebras $A_{Z}$ and $C$ are not split $(Z / F$ being the discriminant quadratic field extension of $(A, \sigma))$;

(3) $n=4, \operatorname{disc}(\sigma)$ is trivial and the algebras $A, C^{+}, C^{-}$are not split.

Proof. - The case $n=3$ follows from Corollary 5.4 since $D_{3}=A_{3}$. If $n=4$, the variety $B G$ is not stably rational even over the discriminant quadratic field extension $Z / F$ by Theorem 8.5 since $H_{\mathrm{nr}}^{3}\left(B G_{Z}\right)_{\text {norm }} \neq 0$.

Remark 8.7. - Examples of stably non-rational classifying varieties $B G$ with simply connected $G$ of type $D_{n}$ exist for $n=3$ and $n=4$ over every number field $F$.

\section{Appendix A. Invariants of algebraic groups}

\section{A.1. Proof of Proposition 3.1}

(The proof is different from the one in [18].) Let $m: S \times X \rightarrow X$ be the action morphism. For any field extension $L / F$ and every $s \in S(L), x \in X(L)$, we have $\tilde{u}_{L}(s x)=\tilde{u}_{L}(x)$. Now let 
$L=F(S \times X)$. Denote by $\eta \in S(L)$ the image of the generic point of $S$ under the embedding $F(S) \hookrightarrow L$ induced by the projection $p_{1}: S \times X \rightarrow S$ and by $\xi^{\prime} \in X(L)$ the image of the generic point $\xi$ of $X$ under the embedding $F(X) \hookrightarrow L$ induced by the projection $p_{2}: S \times X \rightarrow X$. Then $\eta \xi^{\prime} \in X(L)$ is the image of $\xi$ under the embedding $i: F(X) \hookrightarrow L$ induced by $m$.

Choose a point $x \in X$ of codimension 1 . We need to show that

$$
\partial_{x}\left(\tilde{u}_{F(X)}(\xi)\right)=0 \in M^{d-1}(F(x)) .
$$

Consider the point $y \in S \times X$ of codimension 1 with the closure $S \times \overline{\{x\}}$. Since $S$ acts transitively on $X, m(y)$ is the generic point of $X$. Hence the restriction of the discrete valuation on $L$ associated to the point $y$ is trivial on $i(F(X))$. Therefore, by rule R3c in [13],

$$
\partial_{y}\left(\tilde{u}_{L}\left(\xi^{\prime}\right)\right)=\partial_{y}\left(\tilde{u}_{L}\left(\eta \xi^{\prime}\right)\right)=\partial_{y}\left(i_{*} \tilde{u}_{F(X)}(\xi)\right)=0 \in M^{d-1}(F(y)) .
$$

Let $k: F(x) \rightarrow F(y)$ be the field homomorphism induced by the projection $p_{2}: S \times X \rightarrow X$. By the rule R3a in [13] and (A.2),

$$
k_{*}\left(\partial_{x}\left(\tilde{u}_{F(X)}(\xi)\right)\right)=\partial_{y}\left(p_{2 *}\left(\tilde{u}_{F(X)}(\xi)\right)\right)=\partial_{y}\left(\tilde{u}_{L}\left(\xi^{\prime}\right)\right)=0 \in M^{d-1}(F(y)) .
$$

The field $F(y)$ is isomorphic to $F(x)(S)$. Since the smooth variety $S$ has a rational point, the map

$$
k_{*}: M^{d-1}(F(x)) \longrightarrow M^{d-1}(F(y))
$$

is injective (cf. [11, Lemma 1.3]) and hence (A.1) holds.

\section{A.2. Proof of Theorem 3.2}

LEMMA A.1 (Specialization principle). - Let $x_{1}$ and $x_{2}$ be two points of $X$ such that $x_{2}$ is regular and of codimension 1 in $\overline{\left\{x_{1}\right\}}$. We also consider the $x_{i}$ as a point of $X\left(F\left(x_{i}\right)\right)$. Suppose that for an invariant $u \in \operatorname{Inv}^{d}(G, M)$ we have $\tilde{u}_{F\left(x_{1}\right)}\left(x_{1}\right)=0 \in M^{d}\left(F\left(x_{1}\right)\right)$. Then $\tilde{u}_{F\left(x_{2}\right)}\left(x_{2}\right)=0 \in M^{d}\left(F\left(x_{2}\right)\right)$.

Proof. - Denote by $A$ the local ring of the point $x_{2}$ in the variety $\overline{\left\{x_{1}\right\}}$. By assumption, $A$ is a discrete valuation ring with quotient field $F\left(x_{1}\right)$ and residue field $F\left(x_{2}\right)$. Let $\widetilde{A}$ be the completion of $A$, so that

$$
\widetilde{A} \simeq F\left(x_{2}\right)[[t]]
$$

[23, Ch. VIII, Th. 27]. Denote by $E$ the quotient field of $\widetilde{A}$, the completion of the field $F\left(x_{1}\right)$, thus, $E=F\left(x_{2}\right)((t))$. We have the following diagram of maps induced by natural morphisms

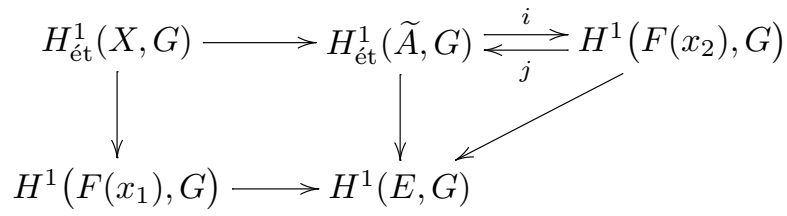

with the bijections $i$ and $j$ inverse to each other [6, Exp. XXIV, Prop. 8.1]. Considering images in all the sets of the diagram of the class in $H_{\text {ét }}^{1}(X, G)$ representing the universal $G$-torsor $S \rightarrow X$, we get

$$
\tilde{u}_{F\left(x_{2}\right)}\left(x_{2}\right)_{E}=\tilde{u}_{E}\left(x_{2}\right)=\tilde{u}_{E}\left(x_{1}\right)=\tilde{u}_{F\left(x_{1}\right)}\left(x_{1}\right)_{E}=0,
$$


i.e., the class $\tilde{u}_{F\left(x_{2}\right)}\left(x_{2}\right)$ splits over $E$. It remains to notice that the map $M^{d}\left(F\left(x_{2}\right)\right) \rightarrow M^{d}(E)$ is injective (being split by a specialization homomorphism [13, p. 329]).

Assume that for $u \in \operatorname{Inv}^{d}(G, M)$ we have $\tilde{u}_{F(X)}(\xi)=0$. For a field extension $L / F$ consider any point $p \in X(L)$, i.e., a morphism $p: \operatorname{Spec}(L) \rightarrow X$. We need to show that $\tilde{u}_{L}(p)=0$. Denote by $x \in X$ the only point in the image of $p$. There is a sequence of points $\xi=x_{1}, x_{2}, \ldots, x_{m}=x$ such that $x_{i+1}$ is regular of codimension 1 in the closure $\overline{\left\{x_{i}\right\}}$ for all $i=1,2, \ldots, m-1$. By Lemma A.1, $\tilde{u}_{F(x)}(x)=0$. The element $p$ is the image of $x$ under $X(F(x)) \rightarrow X(L)$, induced by the natural homomorphism $F(x) \rightarrow L$, hence $\tilde{u}_{L}(p)=0$, being the image of $\tilde{u}_{F(x)}(x)$ under $M^{d}(F(x)) \rightarrow M^{d}(L)$. Thus, $u=0$, i.e., $\theta$ is injective.

Assume now that $S$ is split semisimple simply connected. Let $v \in A^{0}\left(X, M^{d}\right)$ and $x \in X(L)$ be a point over a field extension $L / F$. We define the class $v(x) \in M^{d}(L)$ as the image of $v$ under the pull-back homomorphism

$$
x^{*}: A^{0}\left(X, M^{d}\right) \longrightarrow A^{0}\left(\operatorname{Spec} L, M^{d}\right)=M^{d}(L)
$$

with respect to $x: \operatorname{Spec} L \rightarrow X$. Thus, we get a map

$$
\tilde{u}_{L}: X(L) \longrightarrow M^{d}(L), \quad x \mapsto v(x) .
$$

In order to show that $\tilde{u}_{L}$ defines an invariant $u \in \operatorname{Inv}^{d}(G, M)$ with $\theta(u)=v$ it suffices to prove that the map $v$ is constant on orbits of the $S(L)$-action on $X(L)$.

Let $s \in S(L), x \in X(L)$. Then $v(s x) \in M^{d}(L)$ is the image of $v$ under the pull-back homomorphism with respect to the composition

$$
\operatorname{Spec} L \stackrel{(s, x)}{\longrightarrow} S \times X \stackrel{m}{\longrightarrow} X
$$

where $m$ is the action morphism. The element $v(x) \in M^{d}(L)$ is the image of $u$ under the pullback homomorphism with respect to the composition

$$
\operatorname{Spec} L \stackrel{(s, x)}{\longrightarrow} S \times X \stackrel{p_{2}}{\longrightarrow} X,
$$

where $p_{2}$ is the projection. Thus, it suffices to show that $m$ and $p_{2}$ induce the same homomorphism

$$
m^{*}=p_{2}^{*}: A^{0}\left(X, M^{d}\right) \longrightarrow A^{0}\left(S \times X, M^{d}\right) .
$$

Consider the map $i: X \rightarrow S \times X, i(x)=(1, x)$. Since $p \circ i=\operatorname{id}_{X}=m \circ i$, we have $i^{*} \circ p_{2}^{*}=\mathrm{id}=i^{*} \circ m^{*}$. Hence, it is sufficient to prove that $p_{2}^{*}$ is an isomorphism.

The spectral sequence associated to $p_{2}[13, \S 8]$ :

$$
E_{1}^{p, q}=\coprod_{x \in X^{(p)}} A^{q}\left(S_{F(x)}, M^{d-p}\right) \Rightarrow A^{p+q}\left(S \times X, M^{d}\right)
$$

induces an exact sequence

$$
0 \longrightarrow A^{0}\left(S \times X, M^{d}\right) \stackrel{r}{\longrightarrow} A^{0}\left(S_{F(X)}, M^{d}\right) \stackrel{\partial}{\longrightarrow} \coprod_{x \in X^{(1)}} A^{0}\left(S_{F(x)}, M^{d-1}\right)
$$


The group $S$ is split simply connected, hence, by [7, Th. 4.7(i)], the natural homomorphism

$$
M^{k}(F(x)) \longrightarrow A^{0}\left(S_{F(x)}, M^{k}\right)
$$

is an isomorphism for every $x \in X$ and $k \in \mathbb{Z}$. By [13, Prop. 8.1], the kernel of $\partial$ is isomorphic to $A^{0}\left(X, M^{d}\right)$ and the map $k$ induces an isomorphism

$$
j: A^{0}\left(S \times X, M^{d}\right) \rightarrow A^{0}\left(X, M^{d}\right)
$$

such that the composition $j \circ p_{2}^{*}$ is the identity. Hence $p_{2}^{*}$ is an isomorphism.

COROLlary A.2. - The group $A^{0}\left(X_{\rho}, M^{d}\right)$ does not depend on the choice of an embedding $\rho: G \hookrightarrow S$ into a split semisimple simply connected group $S$.

\section{Appendix B. Rost numbers}

Let $G$ be a split simply connected group defined over a field $F, T \subset G$ a split maximal torus over $F, W$ the Weyl group. The $W$-invariant elements $S^{2}\left(T^{*}\right)^{W}$ in the symmetric square of the character group $T^{*}=\operatorname{Hom}\left(T, \mathbb{G}_{m}\right)$ are $W$-invariant integral quadratic forms on the vector space $V=T_{*} \otimes \mathbb{R}$ of the co-root system, where $T_{*}=\operatorname{Hom}\left(\mathbb{G}_{m}, T\right)$ is the co-character lattice. By [3, Ch. VI, §1, Prop. 7], $S^{2}\left(T^{*}\right)^{W}$ is a free abelian group with a canonical basis given by positive definite forms $q_{1}, q_{2}, \ldots, q_{k}$ corresponding to the $k$ connected components of the Dynkin diagram of $G$. In particular, if $G$ is simple, the group $S^{2}\left(T^{*}\right)^{W}$ is cyclic with the canonical generator $q_{G}$ being a (unique) integral-valued positive definite $W$-invariant quadratic form on $T_{*}$. Since $G$ is simply connected, the lattice of co-characters $T_{*}$ is generated by the co-roots of the root system dual to the root system of $G$. A quadratic form on the space $V$ taking value 1 on short co-roots is integral, hence it coincides with $q_{G}$. Thus, $q_{G}(\beta)=1$ for every short co-root $\beta$.

Example B.1. - Let $G=\mathbf{S L}_{n}, n \geqslant 2$. A split maximal torus $T$ of $G$ is isomorphic to the kernel of the product homomorphism

$$
\left(\mathbb{G}_{m}\right)^{n} \longrightarrow \mathbb{G}_{m} .
$$

Hence the group of co-characters $T_{*}$ can be identified with the subgroup in $\mathbb{Z}^{n}$ consisting of all $n$-tuples $x=\left(x_{1}, x_{2}, \ldots, x_{n}\right)$ with trivial sum of the $x_{i}[9, \S 24]$. The Weyl group $W=S_{n}$ acts by permutations of the $x_{i}$. Clearly, the $W$-invariant integral quadratic form

$$
q_{G}(x)=\frac{1}{2} \sum_{i=1}^{n} x_{i}^{2}=-\sum_{i<j} x_{i} x_{j}
$$

is the canonical generator of $Q(G)$. It takes value 1 on the (short) co-roots $\pm\left(e_{i}-e_{j}\right)$ for $i \neq j$.

Now let $G$ be a (not necessarily split) simply connected group defined over a field $F$. Choose a maximal torus $T \subset G$ over $F$. The absolute $\operatorname{Galois} \operatorname{group} \operatorname{Gal}(F)=\operatorname{Gal}\left(F_{\text {sep }} / F\right)$ acts on $S^{2}\left(T_{\mathrm{sep}}^{*}\right)^{W}$ by permuting the basis forms $q_{i}$ thus $S^{2}\left(T_{\mathrm{sep}}^{*}\right)^{W}$ is a permutation $\mathrm{Gal}(F)$-module. In particular, if $G$ is absolutely simple, the group $S^{2}\left(T_{\mathrm{sep}}^{*}\right)^{W}$ is cyclic with the canonical generator $q_{G}$ and trivial $\mathrm{Gal}(F)$-action. Clearly, the form $q_{G}$ does not change under field extensions.

We denote the group $\left(S^{2}\left(T_{\mathrm{sep}}^{*}\right)^{W}\right)^{\operatorname{Gal}(F)}$ by $Q(G)$. If $G$ is absolutely simple, $Q(G)=\mathbb{Z} q_{G}$.

A homomorphism $\rho: G \rightarrow G^{\prime}$ of simply connected groups induces a homomorphism $Q(\rho): Q\left(G^{\prime}\right) \rightarrow Q(G)[9$, p. 433].

Let $p$ be the characteristic exponent of $F$. 
Theorem B.2 ((Rost) [7, Appendix B, Cor. C.2(b)]). - There is a natural surjective homomorphism

$$
\gamma_{G}: Q(G)[1 / p] \longrightarrow A^{0}\left(B G, H^{3}\right)_{\text {norm }} .
$$

The kernel of $\gamma_{G}$ is generated over $\mathbb{Z}[1 / p]$ by the elements $Q(\alpha)\left(q_{\mathbf{S L}_{n}}\right)$ for all irreducible representations $\alpha: G \rightarrow \mathbf{S L}_{n}$ defined over $F$.

Let $\rho: G \rightarrow G^{\prime}$ be a homomorphism of absolutely simple simply connected groups. Then $Q(\rho)\left(q_{G^{\prime}}\right)=n_{\rho} \cdot q_{G}$ for a uniquely determined integer $n_{\rho} \geqslant 0$. We set

$$
n_{G}=\operatorname{gcd} n_{\alpha}
$$

with the gcd taken over all irreducible representations $\alpha: G \rightarrow \mathbf{S L}_{n}$ of the group $G$. Let $n_{G}^{\prime}$ be the greatest divisor of $n_{G}$ prime to $p$. Thus, $n_{G}=n_{G}^{\prime}$ if $n_{G}$ is relatively prime to $p$.

Denote by $r_{G}$ the element $\gamma\left(q_{G}\right) \in A^{0}\left(B G, H^{3}\right)_{\text {norm. }}$. The corresponding invariant $\theta^{-1}\left(r_{G}\right) \in \operatorname{Inv}^{3}(G, H)_{\text {norm }}$ (Theorem 3.2) we also denote by $r_{G}$ and call it the Rost invariant of $G$.

COROLlARY B.3. - Let $G$ be an absolutely simple simply connected group. Then $A^{0}\left(B G, H^{3}\right)_{\text {norm }}$ is a cyclic group generated by $r_{G}$ of order $n_{G}^{\prime}$.

Note that $r_{G}$ (but not $n_{G}$ ) does not change under field extensions: for a field extension $L / F$, $r_{G_{L}}$ is the image of $r_{G}$ under the canonical homomorphism

$$
A^{0}\left(B G, H^{3}\right)_{\text {norm }} \longrightarrow A^{0}\left(B G_{L}, H^{3}\right)_{\text {norm }} .
$$

An arbitrary simply connected group $G$ is a product of simple simply connected groups $G_{1} \times G_{2} \times \cdots \times G_{k}$. The group $Q(G)$ splits obviously into a direct sum of the $Q\left(G_{i}\right)$. Hence, Theorem B.2 implies

Corollary B.4 ([9, Cor. 31.38]).-

$$
A^{0}\left(B G, H^{3}\right)_{\mathrm{norm}} \simeq \coprod_{i=1}^{k} A^{0}\left(B G_{i}, H^{3}\right)_{\mathrm{norm}} .
$$

Any simple simply connected group $G$ is of the form $R_{L / F}\left(G^{\prime}\right)$, where $L / F$ is a finite separable field extension and $G^{\prime}$ is an absolutely simple simply connected group over $L$. The group $G^{\prime}$ is a canonical direct factor of $G_{L}$, therefore, there are canonical homomorphisms

$$
G_{L} \underset{j}{\stackrel{i}{\gtrless}} G^{\prime}
$$

By naturality of the homomorphism $\gamma_{G}$ in Theorem B.2, the following diagrams commute (with right and left arrows respectively)

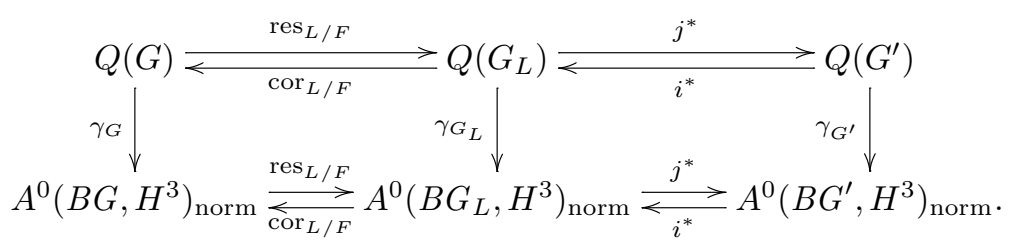


Clearly, the two compositions $j^{*} \circ \operatorname{res}_{L / F}$ and $\operatorname{cor}_{L / F} \circ i^{*}$ in the top row of the diagram are isomorphisms inverse to each other. We have proved

COROLlary B.5 ([9, Cor. 31.39]). - The two compositions $j^{*} \circ \operatorname{res}_{L / F}$ and $\operatorname{cor}_{L / F} \circ i^{*}$ in the bottom row of the diagram

$$
A^{0}\left(B G, H^{3}\right)_{\text {norm }} \rightleftarrows A^{0}\left(B G^{\prime}, H^{3}\right)_{\text {norm }}
$$

are isomorphisms inverse to each other.

\section{B.1. The numbers $n_{\rho}$}

Let $\rho: G \rightarrow G^{\prime}$ be a homomorphism of absolutely simple simply connected groups. Clearly,

$$
n_{G} \mid n_{\rho} \cdot n_{G^{\prime}}
$$

Let $\beta: \mathbb{G}_{m} \rightarrow G$ be a short co-root of $G$. Then

$$
n_{\rho}=n_{\rho} \cdot q_{G}(\beta)=q_{G^{\prime}}(\rho \circ \beta) .
$$

In particular, if $\rho \circ \beta$ is a short co-root of $G^{\prime}$, then $n_{\rho}=1$.

The number $n_{\rho}$ does not change under field extensions. If $\rho^{\prime}: G^{\prime} \rightarrow G^{\prime \prime}$ is another homomorphism of absolutely simple simply connected groups, then

$$
n_{\rho^{\prime} \circ \rho}=n_{\rho^{\prime}} \cdot n_{\rho}
$$

Example B.6. - For the standard inclusion $\rho: \mathbf{S L}_{n} \hookrightarrow \mathbf{S L}_{m}(m>n)$ we have $n_{\rho}=1$ since the co-roots of $\mathbf{S L}_{n}$ are also co-roots of $\mathbf{S L}_{m}$ and have the same length.

Example B.7. - Let $\rho: \mathbf{S p}_{2 n} \hookrightarrow \mathbf{S L}_{2 n}$ be the standard embedding. The embedding of maximal tori is defined by

$$
\left(t_{1}, t_{2}, \ldots, t_{n}\right) \mapsto\left(t_{1}, t_{2}, \ldots, t_{n}, t_{1}^{-1}, t_{2}^{-1}, \ldots, t_{n}^{-1}\right) .
$$

Hence, the map of co-character groups takes $\left(x_{1}, x_{2}, \ldots, x_{n}\right)$ to

$$
\left(x_{1}, x_{2}, \ldots, x_{n},-x_{1},-x_{2}, \ldots,-x_{n}\right) \text {. }
$$

The image of the short co-root $\pm e_{i}$ is the short co-root $\pm\left(e_{i}-e_{n+i}\right)$, hence $n_{\rho}=1$.

Example B.8. - Let $\rho: \mathbf{S p i n}_{2 n} \hookrightarrow \mathbf{S p i n}_{2 n+1}, n \geqslant 3$, be the standard embedding. A maximal torus of the first group is also maximal in the second. The short co-roots $\pm e_{i} \pm e_{j}$ of $G^{\prime}$ correspond to the same short co-roots of $G$, hence $n_{\rho}=1$.

Example B.9. - Let $\rho: \mathbf{S p i n}_{2 n+1} \hookrightarrow \mathbf{S p i n}_{2 n+2}, n \geqslant 2$, be the standard embedding. The homomorphism of co-character groups of maximal tori is induced by the canonical inclusion $\mathbb{Z}^{n} \hookrightarrow \mathbb{Z}^{n+1}$. The co-characters $\pm e_{i} \pm e_{j}$ are the short co-roots in both the groups, hence $n_{\rho}=1$.

Example B.10. - Let $\rho: \mathbf{S p i n}_{n} \hookrightarrow \mathbf{S p i n}_{m}$ be the standard embedding, $m>n \geqslant 5$. By Examples B.8 and B.9, $n_{\rho}=1$. 
Example B.11. - Let $\rho$ be the composition

$$
\operatorname{Spin}_{2 n} \stackrel{\alpha}{\longrightarrow} \mathbf{O}_{2 n}^{+} \stackrel{\longrightarrow}{\longrightarrow} \mathbf{S L}_{2 n}
$$

where $\alpha$ is the standard isogeny. The co-character group of the maximal torus of $\mathbf{S p i n}_{2 n}$ is contained in $\mathbb{Z}^{n}$ with the co-roots $\pm e_{i} \pm e_{j}$ of the same length. The corresponding co-character of the maximal torus of $\mathbf{S L}_{2 n}$ is $\pm\left(f_{i}-g_{i}\right) \pm\left(f_{j}-g_{j}\right)$ if we identify the group of all co-characters with a subgroup in $\coprod \mathbb{Z} f_{i} \oplus \coprod \mathbb{Z} g_{i}$. By Example B.1 and formula (B.2), $n_{\rho}=2$.

\section{B.2. The numbers $n_{G}$}

Let $G$ be a simply connected semisimple group defined over a field $F, \alpha: G \rightarrow \mathbf{S L}(V)$ a representation over $F, T \subset G$ a maximal torus defined over $F$. The space $V_{\text {sep }}=V \otimes_{F} F_{\text {sep }}$ splits into a direct sum of one-dimensional eigenspaces with some eigenvalues $\chi_{1}, \chi_{2}, \ldots$, $\chi_{m} \in T_{\mathrm{sep}}^{*}$. Then, by Example B.1 and (B.2),

$$
n_{\alpha}=\frac{1}{2} \sum_{i}\left\langle\chi_{i}, \eta_{G}\right\rangle^{2} \in \mathbb{Z},
$$

where $\eta_{G} \in T_{\mathrm{sep} *}$ is a short co-root of $G$.

For an algebraic group $H$ denote by $R(H)$ the representation ring of $H$. Consider the following additive group homomorphism

$$
\Phi_{G}: R\left(T_{\mathrm{sep}}\right)=\mathbb{Z}\left[T_{\mathrm{sep}}^{*}\right] \longrightarrow \frac{1}{2} \mathbb{Z}, \quad \sum \chi_{i} \mapsto \frac{1}{2} \sum\left\langle\chi_{i}, \eta_{G}\right\rangle^{2} .
$$

Thus, for a representation $\alpha: G \rightarrow \mathbf{S L}(V)$ we have

$$
n_{\alpha}=\Phi_{G}\left(\left.\alpha\right|_{T_{\mathrm{sep}}}\right) .
$$

The Weyl group $W$ of $G_{\text {sep }}$ acts naturally on $R\left(T_{\text {sep }}\right)$. The absolute Galois group $\operatorname{Gal}(F)$ acts on $R\left(T_{\mathrm{sep}}\right)$ through the $*$-action on $T_{\mathrm{sep}}^{*}$ defined in [21,2.3]. The semidirect product $\Delta$ of $W$ and $\operatorname{Gal}(F)$ acts naturally on $R\left(T_{\mathrm{sep}}\right)$.

Denote by $\Lambda^{+} \subset T_{\mathrm{sep}}^{*}$ the cone of dominant characters (with respect to some system of simple roots). The group $\operatorname{Gal}(F)$ leaves $\Lambda^{+}$invariant. The field of definition of a dominant character $\chi \in \Lambda^{+}$, denoted by $F(\chi)$, is the field corresponding to the stabilizer of $\chi$ in $\operatorname{Gal}(F)$ by Galois theory.

Let $C$ be the center of $G$. For a character $\chi \in T_{\mathrm{sep}}^{*}$ denote by $\bar{\chi} \in C_{\mathrm{sep}}^{*}$ its restriction on $C$. For a dominant character $\chi \in \Lambda^{+}$, the field of definition $F(\bar{\chi})$ of $\bar{\chi}$ is contained in $F(\chi)$. Denote by $A_{\bar{\chi}}$ a Tits algebra associated to $\bar{\chi}[9, \S 27],[22, \S 4]$, so that $A_{\bar{\chi}}$ is a central simple algebra over $F(\bar{\chi})$ uniquely determined up to Brauer equivalence over $F(\bar{\chi})$. For every character $\chi \in \Lambda^{+}$set

$$
A_{\chi}=A_{\bar{\chi}} \otimes_{F(\bar{\chi})} F(\chi) .
$$

The algebra $A_{\chi}$ is a central simple over $F(\chi)$. The index of $A_{\chi}$ depends only on the $\operatorname{Gal}(F)$-orbit of $\chi$.

Let $\chi \in \Lambda^{+}$be a dominant character. Denote by $\Delta(\chi) \in R\left(T_{\mathrm{sep}}\right)^{\Delta}$ the sum in $R\left(T_{\mathrm{sep}}\right)=\mathbb{Z}\left[T_{\mathrm{sep}}^{*}\right]$ of all (finitely many) characters in the $\Delta$-orbit of $\chi$.

ThEOREM B.12 ([22, Th. 3.3]). - The restriction homomorphism $R(G) \rightarrow R\left(T_{\mathrm{sep}}\right)$ is an injection. The elements $\Delta(\chi) \cdot \operatorname{ind}\left(A_{\chi}\right)$, for all $\chi \in \Lambda^{+}$, form a $\mathbb{Z}$-basis of $R(G)$. 
The formula (B.3) then implies

COROLLARY B.13. - For a simply connected group $G$,

$$
n_{G}=\underset{\chi \in \Lambda^{+}}{\operatorname{gcd}}\left[\Phi_{G}(\Delta(\chi)) \cdot \operatorname{ind}\left(A_{\chi}\right)\right] .
$$

\section{B.3. Groups of type $A_{n-1}$}

We compute the number $n_{G}$ for a simply connected group $G$ of type $A_{n-1}$ over a field $F$, $n \geqslant 2$. Let $T \subset G$ be a maximal torus defined over $F$. The group of characters $T_{\text {sep }}^{*}$ can be identified with $\mathbb{Z}^{n} / \mathbb{Z}$ (with $\mathbb{Z}$ embedded diagonally) and $T_{\text {sep } *}$ - with the subgroup of $\mathbb{Z}^{n}$ of elements with the zero sum of the components. All the co-roots $\pm\left(e_{i}-e_{j}\right)$ have the same length and we can take $\eta_{G}=e_{1}-e_{2}$. The Weyl group is the symmetric group $S_{n}$ which permutes the $e_{i}$ (see Example B.1). The restriction homomorphism to the center $C$ of $G$

$$
\mathbb{Z}^{n} / \mathbb{Z}=T_{\text {sep }}^{*} \longrightarrow C_{\text {sep }}^{*}=\mathbb{Z} / n \mathbb{Z}
$$

takes $\left(x_{1}, x_{2}, \ldots, x_{n}\right)+\mathbb{Z}$ to $\sum x_{i}+n \mathbb{Z}$.

We choose the set of simple roots $\left\{e_{1}-e_{2}, e_{2}-e_{3}, \ldots, e_{n-1}-e_{n}\right\}$. The corresponding cone of dominant characters $\Lambda^{+}$consists of all $\left(x_{1}, x_{2}, \ldots, x_{n}\right)+\mathbb{Z}$ such that $x_{1} \geqslant x_{2} \geqslant \cdots \geqslant x_{n}$.

Choose a character $\chi \in T_{\mathrm{sep}}^{*}$. Some of the components of $\chi$ may coincide. Let $\chi$ have distinct components (in some order) $a_{1}>a_{2}>\cdots>a_{k}$ which repeat $r_{1}, r_{2}, \ldots, r_{k}$ times respectively, so that $n=\sum r_{i}$. Note that the $a_{i}$ can be modified by adding an integer to all the $a_{i}$. We denote the character $\chi$ by $\left(r_{1}, \ldots, r_{k} ; a_{1}, \ldots, a_{k}\right)$ or simply by $(\mathbf{r}, \mathbf{a})$.

The stabilizer of $\chi=(\mathbf{r}, \mathbf{a})$ in the Weyl group $S_{n}$ is isomorphic to $S_{r_{1}} \times S_{r_{2}} \times \cdots \times S_{r_{k}}$. Hence the number of characters in the $S_{n}$-orbit of $\chi$ is equal to

$$
\frac{n !}{r_{1} ! r_{2} ! \ldots r_{k} !} \text {. }
$$

For a pair of distinct indices $(i, j)$ the number of characters in the $S_{n}$-orbit with first two components $\left(a_{i}, a_{j}\right)$ is equal to

$$
\frac{(n-2) ! r_{i} r_{j}}{r_{1} ! r_{2} ! \ldots r_{k} !}
$$

For such characters $\chi^{\prime}$ we have $\left\langle\chi^{\prime}, \eta_{G}\right\rangle=a_{i}-a_{j}$. Denote by $S_{n}(\chi)$ the sum of characters in $R\left(T_{\mathrm{sep}}\right)$ of the $S_{n}$-orbit of $\chi$. We have the following computation:

$$
\begin{aligned}
\Phi_{G}\left(S_{n}(\chi)\right) & =\frac{1}{2} \sum_{w \in S_{n}}\left\langle w \chi, \eta_{G}\right\rangle^{2} \\
& =\frac{1}{2} \cdot \frac{(n-2) !}{r_{1} ! r_{2} ! \ldots r_{k} !} \sum_{(i, j)} r_{i} r_{j}\left(a_{i}-a_{j}\right)^{2} \\
& =\frac{(n-2) !}{r_{1} ! r_{2} ! \ldots r_{k} !}\left[\left(\sum_{i} r_{i}\right)\left(\sum_{i} r_{i} a_{i}^{2}\right)-\left(\sum_{i} r_{i} a_{i}\right)^{2}\right] \\
& =\frac{(n-2) !}{r_{1} ! r_{2} ! \ldots r_{k} !}\left[n\left(\sum_{i} r_{i} a_{i}^{2}\right)-\left(\sum_{i} r_{i} a_{i}\right)^{2}\right] .
\end{aligned}
$$

Denote this integer by $\left[r_{1}, \ldots, r_{k} ; a_{1}, \ldots, a_{k}\right]$ or simply by $[\mathbf{r}, \mathbf{a}]$. We also set ra $=\sum r_{i} a_{i}$. 


\section{B.3.1. Inner type}

Let $G$ be a simply connected group of inner type $A_{n-1}$, i.e., $G=\mathbf{S L}_{1}(A)$, where $A$ is a central simple algebra of degree $n$ over $F$. We have $\Delta=W=S_{n}$. The Tits algebra of a character $(\mathbf{r}, \mathbf{a})$ is similar to $A^{\otimes \text { ra }}$ by $[9, \S 27 . \mathrm{B}]$. Hence, by Corollary B.13,

$$
n_{G}=\operatorname{gcd}\left([\mathbf{r}, \mathbf{a}] \cdot \operatorname{ind}\left(A^{\otimes \mathbf{r a}}\right)\right),
$$

where the gcd is taken over all $(\mathbf{r}, \mathbf{a})$ such that $\sum r_{i}=n$.

Denote by $v_{p}$ the $p$-adic valuation on $\mathbb{Z}$. For any integer $c \geqslant 0$, let $s_{p}(c)$ be the sum of the digits in the base $p$ expansion of $c$.

Lemma B.14 ([12, Lemma 5.4(a)]). - If $c=c_{1}+c_{2}+\cdots+c_{k}, c_{i} \geqslant 0$, then

$$
v_{p}\left(\frac{c !}{c_{1} ! c_{2} ! \ldots c_{k} !}\right)=\frac{\sum s_{p}\left(c_{i}\right)-s_{p}(c)}{p-1}
$$

LEMMA B.15. - Let p be a prime integer, $n=r_{1}+r_{2}+\cdots+r_{k}, r_{i} \geqslant 0, l=\min v_{p}\left(r_{i}\right)$ and $v_{p}\left(r_{j}\right)=l$ for some $j$. Then

$$
v_{p}\left(\frac{n !}{r_{1} ! r_{2} ! \ldots r_{k} !}\right) \geqslant v_{p}(n)-l
$$

and the equality holds if and only if

$$
s_{p}(n-1)=s_{p}\left(r_{1}\right)+\cdots+s_{p}\left(r_{j}-1\right)+\cdots+s_{p}\left(r_{k}\right) .
$$

Proof. - We have

$$
\frac{n !}{r_{1} ! r_{2} ! \ldots r_{k} !}=\frac{n}{r_{j}} \cdot \frac{(n-1) !}{r_{1} ! \ldots\left(r_{j}-1\right) ! \ldots r_{k} !}
$$

and the second factor of the r.h.s. is integral, whence the inequality. The second statement follows from Lemma B.14 applied to the second factor.

LEMMA B.16. - For every dominant character $(\mathbf{r}, \mathbf{a}), \operatorname{gcd}(n, \mathbf{r a})$ divides $[\mathbf{r}, \mathbf{a}]$.

Proof. - Let $p$ be a prime divisor of $n, l=\min v_{2}\left(r_{i}\right)$. Obviously,

$$
v_{p}\left(n \cdot \sum_{i} r_{i} a_{i}^{2}\right) \geqslant v_{p}(n)+l, \quad v_{p}\left(\mathbf{r a}^{2}\right) \geqslant v_{p}(\mathbf{r a})+l .
$$

By Lemma B.15,

$$
v_{p}\left(\frac{(n-2) !}{r_{1} ! r_{2} ! \ldots r_{k} !}\right) \geqslant-l
$$

Hence,

$$
v_{p}([\mathbf{r}, \mathbf{a}]) \geqslant-l+\min \left(v_{p}(n)+l, v_{p}(\mathbf{r a})+l\right)=\min \left(v_{p}(n), v_{p}(\mathbf{r a})\right) .
$$

THEOREM B.17. $-n_{G}=\exp (A)$. 
Proof. - We prove first that $n_{G}$ divides $\exp (A)$. In view of (B.4), it suffices to show that for every prime integer $p$ there is a dominant character $(\mathbf{r}, \mathbf{a})$ such that

$$
v_{p}\left([\mathbf{r}, \mathbf{a}] \cdot \operatorname{ind}\left(A^{\otimes \mathbf{r a}}\right)\right)=v_{p}(\exp (A)) .
$$

Let $a=v_{p}(\exp (A))$. We have $v_{p}(n) \geqslant a$ since $\exp (A) \mid n$.

Case 1: $v_{p}(n)>a$. Consider the character $(\mathbf{r}, \mathbf{a})=\left(p^{a}, n-p^{a} ; 1,0\right)$. Then

$$
[\mathbf{r}, \mathbf{a}]=\left(\begin{array}{c}
n-2 \\
p^{a}-1
\end{array}\right), \quad \mathbf{r a}=p^{a}, \quad v_{p}\left(\operatorname{ind}\left(A^{\otimes \mathbf{r a}}\right)\right)=0 .
$$

Clearly, $s_{p}(n-2)=s_{p}\left(n-p^{a}-1\right)$. Hence, by Lemma B.14,

$$
v_{p}\left([\mathbf{r}, \mathbf{a}] \cdot \operatorname{ind}\left(A^{\otimes \mathbf{r a}}\right)\right)=\frac{s_{p}\left(p^{a}-1\right)}{p-1}=a=v_{p}(\exp (A)) .
$$

Case 2: $v_{p}(n)=a$. Since $\exp (A)|\operatorname{ind}(A)| n$, it follows that $v_{p}(\operatorname{ind}(A))=a$. Consider the $\operatorname{character}(\mathbf{r}, \mathbf{a})=(1, n-1 ; 1,0)$. We have $[\mathbf{r}, \mathbf{a}]=1=\mathbf{r a}$ and

$$
v_{p}\left([\mathbf{r}, \mathbf{a}] \cdot \operatorname{ind}\left(A^{\otimes \mathbf{r a}}\right)\right)=v_{p}(\operatorname{ind}(A))=a=v_{p}(\exp (A)) .
$$

It remains to prove that $\exp (A)$ divides $[\mathbf{r}, \mathbf{a}] \cdot \operatorname{ind}\left(A^{\otimes \mathbf{r a}}\right)$ for every dominant character $(\mathbf{r}, \mathbf{a})$. By Lemma B.16,

$$
\exp \left(A^{\otimes[\mathbf{r}, \mathbf{a}]}\right) \mid \exp \left(A^{\otimes \operatorname{gcd}(n, \mathbf{r a})}\right)=\exp \left(A^{\otimes \mathbf{r a}}\right)
$$

and hence

$$
\exp (A)\left|[\mathbf{r}, \mathbf{a}] \cdot \exp \left(A^{\otimes[\mathbf{r}, \mathbf{a}]}\right)\right|[\mathbf{r}, \mathbf{a}] \cdot \operatorname{ind}\left(A^{\otimes \mathbf{r a}}\right) .
$$

By [9, Cor. 29.4], $H^{1}(F, G)=F^{\times} / \operatorname{Nrd}\left(A^{\times}\right)$. Consider the normalized invariant $r_{G}^{\prime}$ of $G$ defined by

$$
r_{G}^{\prime}\left(a \operatorname{Nrd}\left(A^{\times}\right)\right)=(a) \cup[A],
$$

where $[A]$ is the class of the algebra $A$ in the group

$$
H^{2}(F, \mathbb{Q} / \mathbb{Z}(1))=\operatorname{Br}(F)[1 / p]
$$

( $p$ is the characteristic exponent of $F$ ). The residue of the value

$$
r_{G}^{\prime}\left(t \operatorname{Nrd}\left(A_{F((t))}^{\times}\right)\right)=(t) \cup\left[A_{F((t))}\right]
$$

equals $[A]$. Hence the order of $r_{G}^{\prime}$ is divisible by $\exp (A)^{\prime}$, the greatest divisor of $\exp (A)$ prime to $p$. It follows from Theorem B.17 that the invariants $r_{G}$ and $r_{G}^{\prime}$ are two generators of $\operatorname{Inv}^{3}(G, H)_{\text {norm. }}$. In particular, any value of the Rost invariant $r_{G}$ over a field $L$ is the cupproduct $(x) \cup\left[A_{L}\right]$ for some $x \in L^{\times}$. It seems plausible that $r_{G}$ coincides with $r_{G}^{\prime}$ (up to sign).

\section{B.3.2. Outer type}

Let $G$ be a simply connected group of outer type $A_{n-1}$, i.e., $G=\mathbf{S U}(B, \tau)$, where $B$ is a central simple algebra of degree $n \geqslant 3$ with a unitary involution $\tau$ over a separable quadratic 
field extension $K / F$. Over the quadratic extension $K / F$ the group $G$ is isomorphic to $\mathbf{S L}_{1}(B)$. Under the field extension map the Rost invariant $r_{G}$ maps to the Rost invariant $r_{G_{K}}$ of order $\exp (B)$ by Theorem B.17. The corestriction map for the field extension $K / F$ takes $r_{G_{K}}$ to $2 r_{G}$. Hence

$$
\exp (B)\left|n_{G}\right| 2 \exp (B)
$$

B.3.3. Consider first the case when $B$ splits, i.e., $G=\mathbf{S U}(V, h)$, where $(V, h)$ is a nondegenerate hermitian form over $K / F$ of dimension $n$. Let $(V, \bar{h})$ be the associated quadratic form over $F$ of dimension $2 n$. The natural homomorphism $G \rightarrow \operatorname{Spin}(V, \bar{h})$ together with the Arason invariant give a non-trivial invariant of $G$ (see [9, Example 31.44]). Hence $n_{G}=2$ by (B.5).

B.3.4. Assume now that the exponent of $B$ is odd. By (B.3.3), over a field extension of $F$, which splits $B$ (but not $K$ ), the number $n_{G}$ is equal to 2 . Hence $n_{G}$ is even and $n_{G}=2 \exp (B)$ by (B.5). (The Rost invariant in this case is considered in [9, Example 31.45].)

B.3.5. Consider now the general case. We may assume that $n$ is even and set $m=n / 2$. The problem is to decide whether $n_{G}=\exp (B)$ or $n_{G}=2 \exp (B)$. Thus, it is sufficient to trace only the 2-part of these integers.

The Galois group $\operatorname{Gal}(F)$ acts on $T_{\text {sep }}^{*}$ through $\operatorname{Gal}(K / F)$ by the involution

$$
\kappa:\left(x_{1}, x_{2}, \ldots, x_{n}\right)+\mathbb{Z} \mapsto\left(-x_{n}, \ldots,-x_{2},-x_{1}\right)+\mathbb{Z} .
$$

A dominant character $\chi=(\mathbf{r}, \mathbf{a}) \in \Lambda^{+}$is called symmetric if it does not change under $\kappa$, that is, the sequence $\left(r_{1}, r_{2}, \ldots, r_{k}\right)$ is symmetric and the sum $a_{i}+a_{k+1-i}$ does not depend on $i$.

Let $\chi \in \Lambda^{+}$be a dominant character. If $\chi$ is symmetric, then $\Delta(\chi)=W(\chi)$. Otherwise, the $\Delta$-orbit of $\chi$ is twice longer than the $W$-orbit of $\chi$. It is also clear that if $\left(\mathbf{r}^{\prime}, \mathbf{a}^{\prime}\right)=\kappa(\mathbf{r}, \mathbf{a})$, then $\left[\mathbf{r}^{\prime}, \mathbf{a}^{\prime}\right]=[\mathbf{r}, \mathbf{a}]$.

If $\chi=(\mathbf{r}, \mathbf{a})$ is symmetric, then ra is divisible by $m$. The corresponding Tits algebra $A_{\chi}$ is equivalent to $D^{\otimes \frac{\mathrm{ra}}{m}}$, where $D$ is the discriminant algebra of $(B, \tau)$. If $\chi=(\mathbf{r}, \mathbf{a})$ is not symmetric, then the Tits algebra $A_{\chi}$ is equivalent to $B^{\otimes \text { ra }}$ by $[9, \S 27 . \mathrm{B}]$.

It follows from Corollary B.13 that $n_{G}$ is the gcd of two integers $n_{G}^{\prime}$ and $n_{G}^{\prime \prime}$ :

$$
n_{G}^{\prime}=2 \operatorname{gcd}\left([\mathbf{r}, \mathbf{a}] \cdot \operatorname{ind}\left(B^{\otimes \mathbf{r a}}\right)\right),
$$

where the gcd is taken over all non-symmetric dominant characters $(\mathbf{r}, \mathbf{a})$ and

$$
n_{G}^{\prime \prime}=\operatorname{gcd}\left([\mathbf{r}, \mathbf{a}] \cdot \operatorname{ind}\left(D^{\otimes \frac{\mathbf{r a}}{m}}\right)\right),
$$

where the gcd is taken over all symmetric characters $(\mathbf{r}, \mathbf{a})$.

Note that the algebra $D^{\otimes \frac{\mathrm{ra}}{m}} \otimes_{F} K$ is similar to $B^{\otimes \mathbf{r a}}$ [9, Prop. 10.30], hence

$$
\operatorname{ind}\left(D^{\otimes \frac{\mathbf{r a}}{m}}\right) \mid 2 \operatorname{ind}\left(B^{\otimes \mathbf{r a}}\right) .
$$

Therefore, we can modify the integer $n_{G}^{\prime}$ by including in the gcd also symmetric characters, without changing the gcd of $n_{G}^{\prime}$ and $n_{G}^{\prime \prime}$. It follows from (B.4) and Theorem B.17 (applied to the algebra $B$ instead of $A$ ) that $n_{G}^{\prime}=2 \exp (B)$. We get

$$
n_{G}=\operatorname{gcd}\left[2 \exp (B), \operatorname{gcd}\left([\mathbf{r}, \mathbf{a}] \cdot \operatorname{ind}\left(D^{\otimes \frac{\mathbf{r a}}{m}}\right)\right)\right]
$$


where the gcd inside the brackets is taken over all symmetric characters $(\mathbf{r}, \mathbf{a})$. Finally,

$$
n_{G}= \begin{cases}2 \exp (B) & \text { if } 2 \exp (B) \mid[\mathbf{r}, \mathbf{a}] \cdot \operatorname{ind}\left(D^{\otimes \frac{\mathbf{r a}}{m}}\right) \\ & \text { for all symmetric characters }(\mathbf{r}, \mathbf{a}), \\ \exp (B) & \text { otherwise }\end{cases}
$$

Thus, we need to consider divisibility properties of the integers $[\mathbf{r}, \mathbf{a}] \cdot \operatorname{ind}\left(D^{\otimes \frac{\mathbf{r a}}{m}}\right)$ for all symmetric characters $[\mathbf{r}, \mathbf{a}]$. We need only to look at the 2-part of these integers.

Let $\chi=(\mathbf{r}, \mathbf{a})$ be a symmetric character. We consider two cases.

Case 1: The integer $\frac{\mathbf{r a}}{m}$ is even, i.e., ra is divisible by $n$.

We will show (Proposition B.18) that $[\mathbf{r}, \mathbf{a}]$ is 2 -divisible by $2 n$ and hence by $2 \exp (B)$, i.e., the term $[\mathbf{r}, \mathbf{a}] \cdot \operatorname{ind}\left(D^{\otimes \frac{\mathbf{r a}}{m}}\right)$ does not contribute to the $\operatorname{gcd}$.

Proposition B.18. - If $n$ is even, ra is divisible by $n$, then $v_{2}([\mathbf{r}, \mathbf{a}]) \geqslant v_{2}(n)+1$.

Proof. - Let ra $=n q$ for some $q$. We have

$$
[\mathbf{r}, \mathbf{a}]=\frac{1}{n-1} \cdot \frac{n !}{r_{1} ! r_{2} ! \ldots r_{k} !} \cdot\left(\sum r_{i} a_{i}^{2}-n q^{2}\right) .
$$

Since $n-1$ is odd, by Lemma B.15, it suffices to prove that

$$
v_{2}\left(\sum r_{i} a_{i}^{2}-n q^{2}\right) \geqslant l+1
$$

where $l=\min v_{2}\left(r_{i}\right)$. We have

$$
\sum r_{i} a_{i}^{2}-n q^{2}=\sum r_{i} a_{i}\left(a_{i}+1\right)-n q(q+1) .
$$

Since $v_{2}\left(r_{i}\right) \geqslant l$ and $v_{2}(n) \geqslant l$, the r.h.s. is divisible by $2^{l+1}$.

Case 2: The integer $\frac{\text { ra }}{m}$ is odd.

Proposition B.19. - If $n$ is even, $\mathbf{r a}=m q$ with odd $q$, then $v_{2}([\mathbf{r}, \mathbf{a}]) \geqslant v_{2}(m)$. The equality holds if and only if $n$ is a 2-power, $k=2$ and $\mathbf{r}=(m, m)$.

Proof. - By Lemma B.15,

$$
v_{2}\left(\frac{(n-2) !}{r_{1} ! r_{2} ! \ldots r_{k} !}\right) \geqslant-l
$$

where $l=\min v_{2}\left(r_{i}\right)$. Since $q$ is odd, it follows that $v_{2}(m)=v_{2}(\mathbf{r a}) \geqslant l$. Thus, to prove the inequality it is sufficient to show that

$$
v_{2}\left(n \sum r_{i} a_{i}^{2}-m^{2} q^{2}\right) \geqslant v_{2}(m)+l .
$$

It is obvious since $v_{2}\left(r_{i}\right) \geqslant l$ and $v_{2}(m) \geqslant l$.

If $n$ is a 2-power, $k=2$ and $\mathbf{r}=(m, m), \mathbf{a}=\left(a_{1}, a_{2}\right)$ then

$$
\mathbf{r a}=m a_{1}+m a_{2}=m\left(a_{1}+a_{2}\right)=m q,
$$


hence $a_{1}+a_{2}$ is odd. We have

$$
[\mathbf{r}, \mathbf{a}]=\frac{(2 m-2) !}{(m-1) !^{2}}\left(a_{1}-a_{2}\right)^{2}
$$

and by Lemma B.14, since $a_{1}-a_{2}$ is odd,

$$
v_{2}([\mathbf{r}, \mathbf{a}])=v_{2}\left(\frac{(2 m-2) !}{(m-1) !^{2}}\right)=2 s_{2}(m-1)-s_{2}(2 m-2)=s_{2}(m-1)=v_{2}(m) .
$$

Thus, the equality holds.

Conversely, assume the equality. Then the first part of the proof shows that the equality in (B.6) holds, or equivalently,

$$
v_{2}\left(\frac{n !}{r_{1} ! r_{2} ! \ldots r_{k} !}\right)=v_{2}(n)-l
$$

and hence by Lemma B.15,

$$
s_{2}(n-1)=s_{2}\left(r_{1}\right)+\cdots+s_{2}\left(r_{j}-1\right)+\cdots+s_{2}\left(r_{k}\right),
$$

where $j$ satisfies $v\left(r_{j}\right)=l$. This means that when we consequently add (in any order, in base $2)$ the integers $r_{1}, \ldots, r_{j}-1, \ldots, r_{k}$ we never carry over units. In particular, all these integers are pairwise distinct. Thus, the sequence $\mathbf{r}$ can have at most one pair of equal terms. But the character $(\mathbf{r}, \mathbf{a})$ is symmetric, hence the sequence $\mathbf{r}$ is symmetric. It follows that $k \leqslant 3$. If $k=2$, then $\mathbf{r}=(m, m)$ and $s_{2}(2 m-1)=s_{2}(m)+s_{2}(m-1)$, i.e., when we add $m$ and $m-1$ (in base 2 ) we don't carry over units. It is possible only if $m$ (and hence $n$ ) is a 2-power.

Finally, assume $k=3$, i.e., $\mathbf{r}=\left(r_{1}, r_{2}, r_{3}\right)$ with $r_{1}=r_{3}$. Then by symmetry, $a_{1}+a_{3}=2 a_{2}$, hence

$$
m q=r_{1} a_{1}+r_{2} a_{2}+r_{3} a_{3}=r_{1}\left(a_{1}+a_{3}\right)+r_{2} a_{2}=\left(2 r_{1}+r_{2}\right) a_{2}=n a_{2},
$$

therefore, $q=2 a_{2}$, a contradiction since $q$ is odd. This case does not occur.

THEOREM B.20. - Let $G=\mathbf{S U}(B, \tau)$, where $B$ is a central simple algebra of degree $n$ with a unitary involution $\tau$ over a separable quadratic field extension $K / F$. Let $D=D(B, \tau)$ be the discriminant algebra of $(B, \tau)$. Then

$$
n_{G}= \begin{cases}\exp (B) & \text { if } n \text { is a } 2 \text {-power and } \exp (B)=n \\ \exp (B) & \text { if } n \text { is a } 2 \text {-power, } \exp (B)=n / 2, \text { and } D \text { is split } \\ 2 \exp (B) & \text { otherwise. }\end{cases}
$$

Proof. - By (B.5) we may assume that $n$ is even. We know from the cases 1 and 2 considered above that $n_{G}=\exp (B)$ if and only if there exists a symmetric character $(\mathbf{r}, \mathbf{a})$ such that ra $=m q$ with $q$ odd and

$$
v_{2}([\mathbf{r}, \mathbf{a}] \cdot \operatorname{ind}(D))=v_{2}(\exp (B)) .
$$

By Proposition B.19, for such a character $(\mathbf{r}, \mathbf{a})$,

$$
v_{2}([\mathbf{r}, \mathbf{a}]) \geqslant v_{2}(m)
$$


hence equality (B.7) implies

$$
v_{2}(m)+1=v_{2}(n) \geqslant v_{2}(\exp (B)) \geqslant v_{2}(m)+v_{2}(\operatorname{ind}(D)) \geqslant v_{2}(m) .
$$

There are two cases:

Case 1: $v_{2}(\exp (B))=v_{2}(n)=v_{2}(m)+1$.

The algebra $D_{K}$ is similar to $B^{\otimes m}$ and hence is not split. Index ind $(D)$ divides 4 [9, Prop. 10.30], therefore, $\operatorname{ind}(D)$ is even. It follows then from (B.7) that

$$
v_{2}([\mathbf{r}, \mathbf{a}])=v_{2}(\exp (B))-v_{2}(\operatorname{ind}(D)) \leqslant v_{2}(\exp (B))-1=v_{2}(m),
$$

i.e., we have equality in (B.8). By Proposition B.19, $n$ is a 2-power and hence $\exp (B)=n$. Conversely, if $n$ is a 2-power, $\exp (B)=n$, then $D$ has index 2 by [9, Prop. 10.30]. It follows from Proposition B.19 that for the character $(\mathbf{r}, \mathbf{a})=(m, m ; 1,0)$ we have

$$
v_{2}([\mathbf{r}, \mathbf{a}] \cdot \operatorname{ind}(D))=v_{2}([\mathbf{r}, \mathbf{a}])+1=v_{2}(m)+1=v_{2}(n)=v_{2}(\exp (B)),
$$

i.e., (B.7) holds.

Case 2: $v_{2}(\exp (B))=v_{2}(m)$. Comparing (B.7) and (B.8), we deduce that $D$ splits and the equality in (B.8) holds. Hence again by Proposition B.19, $n$ is a 2-power and $\exp (B)=m=$ $n / 2$. Conversely, if $n$ is a 2-power, $\exp (B)=n / 2$ and $D$ is split, then for the same character $(\mathbf{r}, \mathbf{a})$ as in case 1 , by Proposition B.19,

$$
v_{2}([\mathbf{r}, \mathbf{a}] \cdot \operatorname{ind}(D))=v_{2}([\mathbf{r}, \mathbf{a}])=v_{2}(m)=v_{2}(\exp (B))
$$

i.e., (B.7) holds.

Remark B.21. - Inspection of the proof shows that the only source of reduction of the value of $n_{G}$ from $2 \exp (B)$ to $\exp (B)$ is the divisibility property (B.1) for the canonical representation $\rho: G \rightarrow G^{\prime}=\mathbf{S L}_{1}(D)$.

\section{B.4. Groups of type $B_{n}$}

Let $G$ be a simply connected group of type $B_{n}, n \geqslant 1$, i.e., $G=\operatorname{Spin}(V, q)$ for a nondegenerate quadratic form $(V, q)$ of dimension $2 n+1$. Consider the composition

$$
\alpha: \operatorname{Spin}(V, q) \longrightarrow \mathbf{O}^{+}(V, q) \longleftrightarrow \mathbf{S L}(V) .
$$

Since $n_{\alpha}=2$ (Example B.11), we have $n_{G} \mid 2$.

THEOREM B.22. - Let $G$ be a simply connected group of type $B_{n}, n \geqslant 1$, i.e., $G=$ $\operatorname{Spin}(V, q)$ for a non-degenerate quadratic form $(V, q)$ of dimension $2 n+1$. Then

$$
n_{G}= \begin{cases}1 & \text { if } n=1 \text { or } 2 \text { and } q \text { has maximal Witt index } n \\ 2 & \text { otherwise. }\end{cases}
$$

Proof. - The case $n=1$ follows from Theorem B.17 since $G \simeq \mathbf{S L}_{1}\left(C_{0}\right)$, where $C_{0}$ is the even Clifford algebra of $(V, q)$ by [9, Th. 15.2] and $q$ is isotropic if and only if $C_{0}$ is split. If $n=2$ and $q$ is of Witt index 2 , then $G$ splits and hence $G \simeq \mathbf{S p}_{4}$ (since $B_{2}=C_{2}$ ) and the latter group is special, therefore $n_{G}=1$. 
Assume that $n \geqslant 2$ and the Witt index of $q$ is less that 2 if $n=2$. The image of the map $H^{1}(F, G) \rightarrow H^{1}\left(F, \mathbf{O}^{+}(V, q)\right)$ classifies quadratic forms $q^{\prime}$ on $V$ such that $q \perp-q^{\prime} \in I^{3}$ (cf. $[9,31.41])$. The invariant taking $q^{\prime}$ to the Arason invariant of $q \perp-q^{\prime}$ is non-trivial, hence it coincides with $r_{G}$ and therefore $n_{G}=2$.

\section{B.5. Groups of type $C_{n}$}

Let $G$ be a simply connected group of type $C_{n}, n \geqslant 1$, i.e., $G=\mathbf{S p}(A, \sigma)$ for a central simple algebra $A$ of degree $2 n$ with a symplectic involution $\sigma$. Let

$$
\alpha: \operatorname{Sp}(A, \sigma) \longleftrightarrow \mathbf{S L}_{1}(A)
$$

be the natural embedding. Since $n_{\alpha}=1$ (Example B.7), it follows from Theorem B.17 and (B.1) that

$$
n_{G} \mid n_{\alpha} \cdot n_{\mathbf{S L}_{1}(A)}=\exp (A) .
$$

In the case $A$ splits we have then $n_{G}=1$, and in general, $n_{G} \mid 2$ since $\exp (A) \mid 2$.

THEOREM B.23. - Let $G$ be a simply connected group of type $C_{n}, n \geqslant 1$, i.e., $G=\mathbf{S p}(A, \sigma)$ for a central simple algebra A of degree $2 n$ with a symplectic involution $\sigma$. Then

$$
n_{G}= \begin{cases}1 & \text { if A splits, } \\ 2 & \text { otherwise } .\end{cases}
$$

Proof. - We may assume that $A$ is not split. Suppose first that $A$ is similar to a quaternion division algebra $Q$. By [9, Th. 4.2], the involution $\sigma$ is adjoint to a hermitian form $(V, h)$ of rank $n$ over $Q$ with respect to the canonical involution on $Q$. Let $\left(V_{0}, h_{0}\right)$ be a non-degenerate subform of $h$ of rank 1 . We have an embedding

$$
\beta: \mathbf{S L}_{1}(Q)=\mathbf{S p}\left(V_{0}, h_{0}\right) \hookrightarrow \mathbf{S p}(V, h)=G
$$

with $n_{\beta}=1$ (Example B.7) and $2=n_{\mathbf{S L}_{1}(Q)} \mid n_{\beta} \cdot n_{G}=n_{G}$ by Theorem B.17 and (B.1), hence $n_{G}=2$.

In general, choose a field extension $L / F$ such that $A_{L}$ is similar to a quaternion algebra. Since $2=n_{G_{L}} \mid n_{G}$, it follows that $n_{G}=2$.

\section{B.6. Groups of type $D_{n}$}

Let $G$ be a simply connected group of (classical) type $D_{n}, n \geqslant 4$, i.e., $G=\operatorname{Spin}(A, \sigma, f)$ for a central simple algebra $A$ of degree $2 n$ with a quadratic pair $(\sigma, f)(\operatorname{simply} G=\operatorname{Spin}(A, \sigma)$ if char $F \neq 2$ ). Consider the composition

$$
\rho: \operatorname{Spin}(A, \sigma, f) \stackrel{\alpha}{\longrightarrow} \mathbf{O}^{+}(A, \sigma, f) \longleftrightarrow \mathbf{S L}_{1}(A)
$$

where $\alpha$ is the standard isogeny. Since by Example B.11, $n_{\rho}=2$, it follows from Theorem B.17 and (B.1) that

$$
n_{G} \mid n_{\alpha} \cdot n_{\mathbf{S L}_{1}(A)}=2 \exp (A) .
$$

In the case $A$ splits it implies then $n_{G} \mid 2$, and in general, $n_{G} \mid 4$ since $\exp (A) \mid 2$.

If $A$ splits, i.e., $G=\operatorname{Spin}(V, q)$ for a quadratic form $(V, q)$ of dimension $2 n \geqslant 8$, there is a non-trivial Arason invariant, hence $n_{G}=2$. 
Let $Z / F$ be the discriminant quadratic extension (the center of the Clifford algebra $C(A, \sigma, f))$. It is an étale quadratic extension of $F$.

The character group $T_{\text {sep }}^{*}$ can be identified with $\mathbb{Z}^{n}+\mathbb{Z} \varepsilon$, where

$$
\varepsilon=\frac{e_{1}+e_{2}+\cdots+e_{n}}{2} .
$$

The group of co-characters $T_{\mathrm{sep} *}$ is identified with the subgroup in $\mathbb{Z}^{n}$ of the elements with even sum of the components. All the co-roots $\pm e_{i} \pm e_{j}$ have the same length and we can take $\eta_{G}=e_{1}-e_{2}$. The Weyl group $W$ is a semidirect product of $H=(\mathbb{Z} / 2 \mathbb{Z})^{n-1}$ and the symmetric group $S_{n}$ : the elements of $H$ change signs in even number of places and $S_{n}$ permutes the $e_{i}$. The Galois group $\operatorname{Gal}(F)$ acts on $T_{\text {sep }}^{*}$ through $\operatorname{Gal}(Z / F)$ by the involution

$$
\kappa:\left(x_{1}, \ldots, x_{n-1}, x_{n}\right)+\mathbb{Z} \mapsto\left(x_{1}, \ldots, x_{n-1},-x_{n}\right)+\mathbb{Z} .
$$

We choose the set of simple roots $e_{1}-e_{2}, e_{2}-e_{3}, \ldots, e_{n-1}-e_{n}, e_{n-1}+e_{n}$. The corresponding cone of dominant characters $\Lambda^{+}$consists of all characters $\left(x_{1}, x_{2}, \ldots, x_{n}\right)$ such that $x_{1} \geqslant x_{2} \geqslant \cdots \geqslant x_{n-1} \geqslant\left|x_{n}\right|$.

Let $C$ be the center of $G$. The group $C^{*}$ consists of 4 elements: $0, \lambda, \lambda^{+}$and $\lambda^{-}$, where $\lambda$ is trivial on the kernel of the isogeny $\alpha$. The corresponding Tits algebra $A_{\lambda}$ is similar to $A$ [9, 27.B]. The restriction of $\bar{\chi}$ of a character $\chi=\left(x_{1}, x_{2}, \ldots, x_{n}\right)$ to $C$ satisfies

$$
\bar{\chi}= \begin{cases}0 & \text { if all the } x_{i} \text { are integers and } \sum x_{i} \text { is even } \\ \lambda & \text { if all the } x_{i} \text { are integers and } \sum x_{i} \text { is odd } \\ \lambda^{+} \text {or } \lambda^{-} & \text {if all the } x_{i} \text { are semi-integers. }\end{cases}
$$

\section{B.6.1. Inner case}

Assume that $Z$ splits. Then $C(A, \sigma, f)=C^{+} \times C^{-}$, where $C^{+}$and $C^{-}$are central simple algebras over $F$ being Tits algebras of $\lambda^{+}$and $\lambda^{-}$. Denote by $n_{0}, n_{1}, n^{+}$and $n^{-}$the $\operatorname{gcd} \Phi_{G}(W(\chi))$ for all dominant characters $\chi$ restricting to $0, \lambda, \lambda^{+}$and $\lambda^{-}$respectively. We have

$$
n_{G}=\operatorname{gcd}\left(n_{0}, n_{1} \cdot \operatorname{ind}(A), n^{+} \cdot \operatorname{ind}\left(C^{+}\right), n^{-} \cdot \operatorname{ind}\left(C^{-}\right)\right) .
$$

Consider a dominant character $\chi=\left(x_{1}, \ldots, x_{n}\right)$ with integer components. Assume first that only one of the components is nonzero, i.e., $\chi=(a, 0, \ldots, 0)=a e_{1}$ with $a>0$. The $W$-orbit of $\chi$ consists of the characters $\pm a e_{i}$, hence $\Phi_{G}(W(\chi))=2 a^{2}$. In particular, $n_{1} \mid 2$.

Assume now that $\chi$ has at least two nonzero components, i.e., $\chi=(a, b, \ldots)$ with $a>b>0$. We claim that $\Phi_{G}(W(\chi))$ is divisible by 4 . Consider the subgroup $W^{\prime} \subset \mathrm{W}$ being the semidirect product of $H$ and $S_{2}$ interchanging the first two components. It suffices to show that $\Phi_{G}\left(W^{\prime}\left(\chi^{\prime}\right)\right)$ is divisible by 4 for every $\chi^{\prime} \in W \chi$. Each orbit $W^{\prime} \chi^{\prime}$ is the union of the following sets of characters:

- $( \pm c, \pm d, \ldots)$ and $( \pm d, \pm c, \ldots)$ for nonzero $c \neq d$;

- $( \pm c, \pm c, \ldots)$;

- $( \pm c, 0, \ldots, \pm d, \ldots)$ and $(0, \pm c, \ldots, \pm d, \ldots)$ for nonzero $c, d$.

One easily checks that the value $\Phi_{G}$ of the sum of characters in each set is divisible by 4 . We have proved

LEMMA B.24. - The integer $n_{0}$ is divisible by 4 and $n_{1}=2$.

Now consider the integers $n^{+}$and $n^{-}$. All the coordinates of a character $\chi$ restricting to $\lambda^{+}$ or $\lambda^{-}$are semi-integers, and in particular are nonzero. 
LEMMA B.25. $-n^{+}=n^{-}=2^{n-3}$.

Proof. - Clearly, $\Phi_{G}(W(\varepsilon))=2^{n-3}$. We claim that $\Phi_{G}(W(\chi))$ is divisible by $2^{n-3}$ for every character $\chi$ with semi-integer components. It suffices to show that $\Phi_{G}\left(W^{\prime}(\chi)\right)$ is divisible by $2^{n-3}$ for every character $\chi$ with semi-integer components. We split the orbit $W^{\prime} \chi$ into a union of the pairs $\chi_{1}=a e_{1}+b e_{2}+\cdots, \chi_{2}=-a e_{1}+b e_{2}+\cdots$ with semi-integers $a$ and $b$. Then

$$
\Phi_{G}\left(\chi_{1}+\chi_{2}\right)=2\left(a^{2}+b^{2}\right) \in \frac{1}{2} \mathbb{Z}
$$

and the number of pairs in the orbit is $2^{n-2}$, whence the claim.

Lemmas B.24 and B.25 and (B.9) give then the following theorem.

THEOREM B.26. - Let $G$ be a simply connected group of classical type $D_{n}, n \geqslant 4$, i.e., $G=\operatorname{Spin}(A, \sigma, f)$ for a central simple algebra $A$ of degree $2 n$ with a quadratic pair $(\sigma, f)$. If $\operatorname{disc}(\sigma, f)$ is trivial,

$$
n_{G}= \begin{cases}2 & \text { if } A \text { splits } \\ 2 & \text { if } n=4 \text { and one of the algebras } C^{+} \text {and } C^{-} \text {splits } \\ 4 & \text { otherwise }\end{cases}
$$

\section{B.6.2. Outer case}

The group $\Delta$ is a semidirect product of $(\mathbb{Z} / 2 \mathbb{Z})^{n}$ and $S_{n}$.

THEOREM B.27. - Let $G$ be a simply connected group of classical type $D_{n}, n \geqslant 4$, i.e., $G=\operatorname{Spin}(A, \sigma, f)$ for a central simple algebra $A$ of degree $2 n$ with a quadratic pair $(\sigma, f)$. If $\operatorname{disc}(\sigma, f)$ is nontrivial,

$$
n_{G}= \begin{cases}2 & \text { if A splits } \\ 4 & \text { otherwise. }\end{cases}
$$

Proof. - As in the inner case we prove that $n_{G} \mid 2 \exp (A)$. If $A$ splits, nontriviality of the Arason invariant implies that $n_{G}=2$. Assume that $A$ is not split. It suffices to show that for every character $\chi \in \Lambda^{+}$, the integer

$$
\Phi_{G}(\Delta(\chi)) \cdot \operatorname{ind}\left(A_{\chi}\right)
$$

is divisible by 4 .

Assume first that only one of the components of $\chi$ is nonzero, i.e., $\chi=a e_{1}$ with positive integer $a$. The $\Delta$-orbit of $\chi$ consists of the characters $\pm a e_{i}$, hence $\Phi_{G}(\Delta(\chi))=2 a^{2}$. Note that $\chi$ is stable under the involution $\kappa$, hence $F(\chi)=F$ and $A_{\chi}=A^{\otimes a}[9,27$. A]. If $a$ is odd, then the algebra $A^{\otimes a}$ does not split, $\operatorname{ind}\left(A^{\otimes a}\right)$ is even and hence the integer (B.10) is divisible by 4 .

If $\chi$ has at least two nonzero components, then as in the inner case we see that even $\Phi_{G}(\Delta(\chi))$ is divisible by 4 .

Finally assume that all the components of $\chi$ are semi-integers. The orbit $\Delta \chi$ is twice longer than in the inner case, hence as in the proof of Lemma B.25 we see that $\Phi_{G}(\Delta(\chi))$ is divisible by $2^{n-2}$ and therefore by 4 since $n \geqslant 4$.

\section{REFERENCES}

[1] Blanchet A., Function fields of generalized Brauer-Severy varieties, Comm. Algebra 19 (1991) 97-118. 
[2] Bogomolov F.A., Brauer groups of the fields of invariants of algebraic groups, Mat. Sb. 180 (2) (1989) 279-293.

[3] Bourbaki N., Groupes et Algèbres de Lie, Chap. 4, 5, 6, Masson, Paris, 1981.

[4] Colliot-ThÉlÈne J.-L., S Ansuc J.-J., The rationality problem for fields of invariants under linear algebraic groups (with special regards to the Brauer group), Notes from the 1988 ELAM conference, Santiago de Chile, http://www.math.u-psud.fr/ colliot/liste-publi.html, 1988.

[5] Colliot-Thélène J.-L., Birational invariants purity, and the Gersten conjecture purity, in: Jacob W., Rosenberg A. (Eds.), Proceedings of the 1992 Summer Research Institute on Quadratic Forms and Division Algebras, Symposia Pure Math., Vol. 58.1, American Mathematical Society, Providence, RI, 1995, pp. 1-64.

[6] Demazure M., Grothendieck A., Schémas et Groupes III (SGA 3, Tome III), Lect. Notes in Math., Vol. 153, Springer, Heidelberg, 1970.

[7] Esnault H., Kahn B., Levine M., Viehweg E., The Arason invariant and mod 2 algebraic cycles, J. Amer. Math. Soc. 11 (1) (1998) 73-118.

[8] Garibaldi R., Tignol J.-P., WAdsworth A., Galois cohomology of special orthogonal groups, Manuscripta Math. 93 (2) (1997) 247-266.

[9] Knus M.-A., Merkurjev A.S., Rost M., Tignol J.-P., The Book of Involutions, American Mathematical Society Colloquium Publications, Vol. 44, American Mathematical Society, Providence, RI, 1998.

[10] LAM T.-Y., The Algebraic Theory of Quadratic Forms, Benjamin, Reading, MA, 1973.

[11] MerkurJeV A.S., Invariants of algebraic groups, J. Reine Angew. Math. 508 (1999) 127-156.

[12] MerkurJev A.S., PANin I.A., WAdsworth A., Index reduction formulas for twisted flag varieties, II, K-Theory 14 (2) (1998) 101-196.

[13] Rost M., Chow groups with coefficients, Documenta Mathematica 1 (1996) 319-393.

[14] Saltman D., The Brauer group and the center of generic matrices, J. Algebra 97 (1) (1985) 53-67.

[15] Saltman D., Invariant fields of linear groups and division algebras, in: Perspectives in Ring Theory (Antwerp, 1987), Kluwer Academic, 1988, pp. 279-297.

[16] Saltman D., Brauer groups of invariant fields, geometrically negligible classes, an equivariant Chow group, and unramified $H^{3}$, in: Jacob W., Rosenberg A. (Eds.), Proceedings of the 1992 Summer Research Institute on Quadratic Forms and Division Algebras, Symposia Pure Math., Vol. 58.1, American Mathematical Society, Providence, RI, 1995, pp. 189-246.

[17] Saltman D., The Schur index and Moody's theorem, K-Theory 7 (1993) 309-332.

[18] SERRE J.-P., Letter to M. Rost, Harvard, Dec. 3, 1992.

[19] Serre J.-P., Cohomologie galoisienne, Lect. Notes Math., Vol. 5, Cinquième édition, révisée et complétée, Springer-Verlag, Berlin, 1994.

[20] SERre J.-P., Cohomologie galoisienne: progrès et problèmes, Séminaire Bourbaki, Vol. 1993/94, Astérisque (1995), no. 227, Exp. No. 783, 4, 229-257.

[21] Tits J., Classification of algebraic semisimple groups, in: Borel A., Mostow G.D. (Eds.), Algebraic Groups and Discontinuous Subgroups, Proc. Symp. Pure Math., Vol. 9, 1966, pp. 33-62.

[22] Tits J., Représentations linéaires irréductibles d'un groupe réductif sur un corps quelconque, J. Reine Angew. Math. 247 (1971) 196-220.

[23] Zariski O., SAmuel P., Commutative Algebra. II, Graduate Texts in Mathematics, Vol. 29, SpringerVerlag, 1975.

Alexander MerkuRJeV

Department of Mathematics

University of California,

Los Angeles, CA 90095-1555 USA

E-mail: merkurev@math.ucla.edu 